This item was submitted to Loughborough's Research Repository by the author.

Items in Figshare are protected by copyright, with all rights reserved, unless otherwise indicated.

\title{
Microneedle assisted micro-particle delivery by gene guns: mathematical model formulation and experimental verification
}

\section{PLEASE CITE THE PUBLISHED VERSION}

http://dx.doi.org/10.1016/j.ces.2014.06.031

\section{PUBLISHER}

(C) Elsevier

VERSION

AM (Accepted Manuscript)

\section{PUBLISHER STATEMENT}

This work is made available according to the conditions of the Creative Commons Attribution-NonCommercialNoDerivatives 4.0 International (CC BY-NC-ND 4.0) licence. Full details of this licence are available at: https://creativecommons.org/licenses/by-nc-nd/4.0/

\section{LICENCE}

CC BY-NC-ND 4.0

\section{REPOSITORY RECORD}

Zhang, Dongwei, Diganta Bhusan Das, and Chris D. Rielly. 2014. "Microneedle Assisted Micro-particle Delivery by Gene Guns: Mathematical Model Formulation and Experimental Verification". figshare. https://hdl.handle.net/2134/15549. 
This item was submitted to Loughborough's Institutional Repository (https://dspace.lboro.ac.uk/) by the author and is made available under the following Creative Commons Licence conditions.

\section{creative
commons}

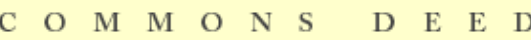

Attribution-NonCommercial-NoDerivs 2.5

You are free:

- to copy, distribute, display, and perform the work

Under the following conditions:

Attribution. You must attribute the work in the manner specified b the author or licensor.

Noncommercial. You may not use this work for commercial purposes.

No Derivative Works. You may not alter, transform, or build upon this work.

- For any reuse or distribution, you must make clear to others the license terms of this work.

- Any of these conditions can be waived if you get permission from the copyright holder.

Your fair use and other rights are in no way affected by the above.

This is a human-readable summary of the Leqal Code (the full license).

\section{Disclaimer 만}

For the full text of this licence, please go to: http://creativecommons.org/licenses/by-nc-nd/2.5/ 


\title{
Microneedle assisted micro-particle delivery by gene guns:
}

Mathematical model formulation and experimental verification

\author{
Dongwei Zhang, Diganta B Das*, Chris D Rielly \\ Department of Chemical Engineering, Loughborough University, Loughborough, LE11 3TU, \\ Leicestershire. UK
}

Short title: Microneedle assisted micro-particle delivery

Submitted for review and publication in:

Chemical Engineering Science

13 June 2014

*Corresponding author; Email: d.b.das@lboro.ac.uk; Tel: 00441509222509 


\title{
Microneedle assisted micro-particle delivery by gene guns: Mathematical model formulation and experimental verification
}

\author{
Dongwei Zhang, Diganta B Das*, Chris D Rielly \\ Department of Chemical Engineering, Loughborough University, Loughborough LE11 3TU, Leicestershire. UK
}

(*Corresponding author; Email: d.b.das@lboro.ac.uk; Tel: 00441509222509)

\section{Abstract}

Gene gun is a micro-particles delivery system which accelerates DNA loaded micro-particles to a high speed so as to enable penetration of the micro-particles into deeper tissues to achieve gene transfection. Previously, microneedle (MN) assisted micro-particles delivery has been shown to achieve the purpose of enhanced penetration depth of micro-particles based on a set of laboratory experiments. In order to further understand the penetration process of micro-particles, a mathematical model for $\mathrm{MN}$ assisted micro-particles delivery is developed. The model mimics the acceleration, separation and deceleration stages of the operation of a gene gun (or experimental rig) aimed at delivering the micro-particles into tissues. The developed model is used to simulate the particle velocity and the trajectories of micro-particles while they penetrate into the target. The model mimics the deceleration stage to predict the linear trajectories of the micro-particles which randomly select the initial positions in the deceleration stage and enter into the target. The penetration depths of the micro-particles are analyzed in relation to a number of parameters, e.g., operating pressure, particle size, and MNs length. Results are validated with experimental results obtained from the previous work. The results also show that the particle penetration depth is increased from an increase of operating pressure, particle size and MN length. The presence of the pierced holes causes a surge in penetration distance.

Key words: Modelling, gene gun, micro-particle, microneedle, penetration depth

\section{Introduction}

Gene guns are aimed at delivering DNA loaded micro-particles into target tissues at high speed (Zhang et al., 2013a; O'Brien et al., 2006; Yang et al., 2004; Lin, 2000). The penetration depths of the micro-particles are typically greater than the stratum corneum, i.e., the top layer of skin (Yager et al., 2013; Kendall et al., 2004a,b; Mitchell et al., 2003; Chen et al., 2002; Quinlan et al., 2001). To understand various features of the micro-particles delivery and evaluate achievable performance from the gene guns, mathematical models are often developed which aim to simulate the micro-particles transfer process for specific gene 
delivery system. For example, Liu (2006) has focused on simulating the velocity distribution in the converging (conical) section of a venturi system developed for a gene gun, namely, the PowderJect system (Bellhouse et al., 1994, 2003, 2006). The particle velocity has been simulated based on a balance between the inertia of micro-particles and other forces acting on the particles. Zhang et al. (2007) have used the programming platform MATrix LABoratory (MATLAB, the MathWorks Inc., Natick, USA; Shampine et al., 1997) to simulate three different stages of the particle delivery from a gene gun, namely, acceleration, separation and deceleration stages. In this work, the particle velocity have been analyzed on the basis of Newton's second law in the acceleration stage, energy conservation is applied to describe the separation of micro-carriers into micro-particles in the separation stage, and Stock's law is used to model the micro-particles penetration in the deceleration stage. Soliman et al. (2011) have used a commercial turbo-machinery flow simulator, namely, FINE ${ }^{\mathrm{TM}}$ /Turbo (NUMECA International, Brussels, Belgium) to simulate the behaviour of gas and particle flow in a supersonic core jet in a gene gun. This work has used Newton's second law to determine the penetration depths of micro-particles in the skin. As discussed below, a number of other studies have shown that the penetration depths of micro-particles depend on the momentums of the particles which again depend on the particle size, density and velocity.

As well known, human skin is a major component of the body that must be considered in the study of micro-particles penetration. The skin helps to prevent the entry of foreign substances into the body (Holbrook et al., 1974; Scheuplein et al., 1971). It also provides a great resistance to the moving micro-particles during a particle delivery process. The skin consists of three main layers, which are the stratum corneum (SC), viable epidermis (VE) and the dermis (Parker, 1991; Phipps et al., 1988). On

55 average the stratum corneum is between 10 and $20 \mu \mathrm{m}$ thick (Holbrook et al., 1974) which may vary in different regions of the body and amongst different groups of people. The thickness of the epidermis also varies in different regions of the body but it has been reported to have an average thickness of 20 to 100 $\mu \mathrm{m}$ (Matteucci et al., 2009; Schaefer et al., 1996). In addition, the thickness of the dermis varies between 1.5 and 3mm (Lambert et al., 2008) and especially on the back it can be up to $4 \mathrm{~mm}$ thick (Rushmer 1966). The VE of the skin is the target layer for of DNA vaccination for previous needle free gene gun systems. 
61

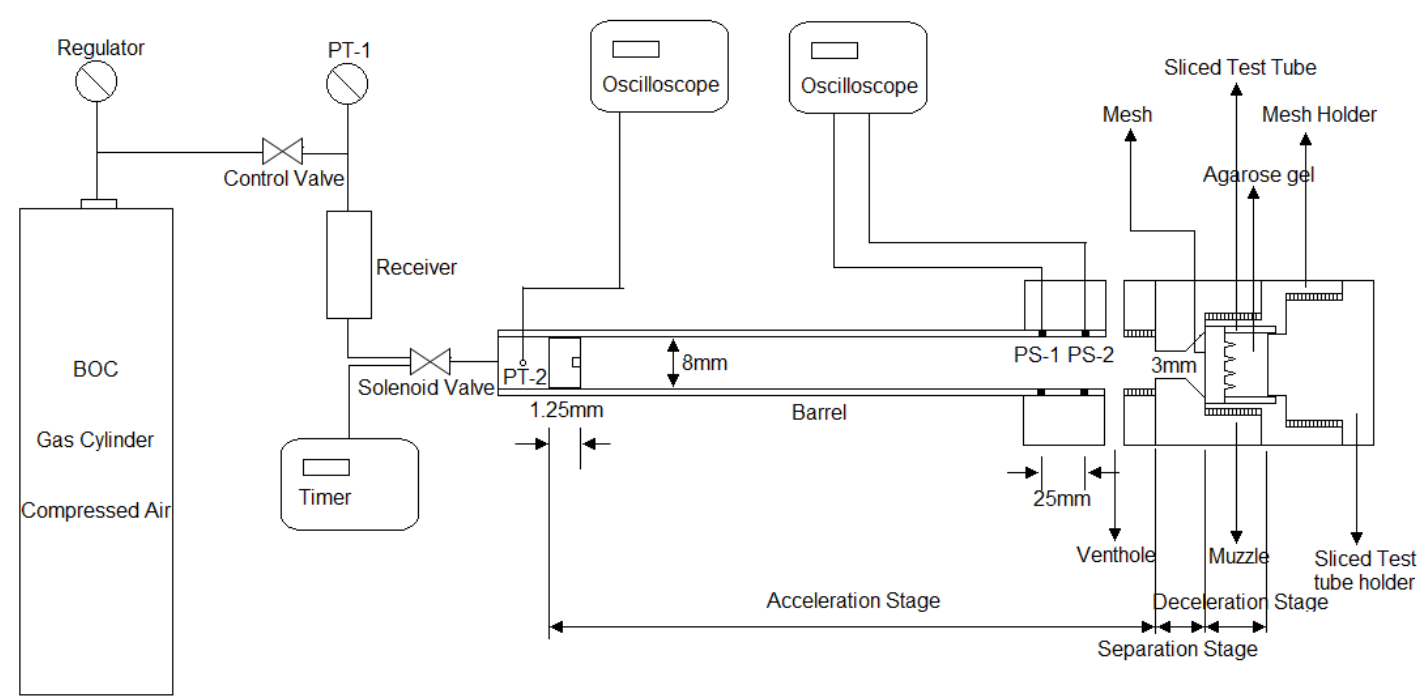

Figure 1: A schematic diagram of the experimental rig for $\mathrm{MN}$ assisted micro-particles delivery (Zhang et al., 2013b)

In a recent study, it has been shown that the penetration depths of micro-particles could be improved further to the dermis layer based on the use of microneedles (MNs) by creating holes on the target which allow a percentage of micro-particles penetrate through to achieve the purpose of improved penetration depth (Zhang et al., 2013b). MN arrays are minimally invasive systems that bypass the outer layer of skin, namely the stratum corneum, to achieve increased transdermal drug delivery (e.g., Olatunji and Das, 2011; Donnelly et al., 2012; Nayak et al., 2013; Olatunji et al., 2013; Cheung et al., 2014). MNs are broadly classified into two categories, namely, solid and hollow (e.g., Al-Qallaf et al., 2009; Olatunji and Das, 2010; Olatunji et al., 2012; Nayak and Das, 2013; Han and Das, 2013; Zhang et al., 2013a,b; Olatunji et al., 2014). Using MNs and gene gun mimicking experimental rig (Figure 1), Zhang et al. (2013b) have shown that pellets bound with $40 \mathrm{mg} / \mathrm{ml}$ concentration of Polyvinylpyrrolidone (PVP) concentration yield approximately $70 \%$ of passage percentage of a pellet mass with good control on the size distribution of separated micro-particles using a mesh of $178 \mu \mathrm{m}$ pore size. Solid micro-needles were used by Zhang et al., 2013 (b) to create pores/holes in the target tissue which remain for sufficiently long time after removing the MN. Within that time, micro-particles can be fired in the same tissue. It has been shown that a number of micro-particles can penetrate into the tissue via the holes while other micro-particles may be stopped from penetrating into the target by the non-porous (i.e., without MNs created holes) area of the tissue. The micro-particles transfer process of this system is divided into three stages, which are the acceleration, separation and deceleration stages. For the acceleration stage, a ground slide carries a pellet of micro-particles which are accelerated together to a desired speed by high pressure compressed air. In the separation stage, the pellet will be released from the ground slide after it reaches a stopping wall in a 
barrel; thereby it separates into micro-particles by a stopping screen with high speed. For the deceleration stage, the separated micro-particles spray forward, penetrate into the target via the holes made by solid micro-needle and stop inside the target.

Zhang et al. (2014) have shown that the penetration depth of stainless steel micro-particles is enhanced in a skin mimicking agarose gel by using MNs. The work uses an agarose powder to prepare an agarose gel of a specific concentration $(2.65 \mathrm{~g} / \mathrm{ml})$ to mimic the porcine skin based on its viscoelastic properties. This skin mimicking agarose gel is considered as a target instead of human skin to analyze the penetration depth of stainless steel micro-particles in relation to the operating pressure, particle size and MN length due to their homogeneity and transparency provide a good environment to observe the penetration by a digital optical microscope. The penetration depth of micro-particles in the gel is analyzed by an image processing software, namely, ImageJ (National Institutes of Health, Maryland, USA) (ImageJ, 2013). Zhang et al. (2014) show that the penetration depth increases with an increase of operating pressure, particle size and $\mathrm{MN}$ length. Especially, the high-speed micro-particles penetrate further into the target via the pierced holes which are created by MNs. However, the maximum penetration depth depends on the MN length which makes different lengths of holes on the target.

102 In order to further understand the characteristics of MNs assisted micro-particles delivery from gene guns, the present study aims to develop a mathematical model of the process (Figure 1) for delivering the micro-particles into a target. In particular this paper aims to simulate the penetration depths of the micro-particles. From an experimental point of view, the micro-particles are compressed into a cylinder pellet, loaded into a ground slide, and accelerated by pressurized air. Thus, the velocity of the pellet is defined to be equal to the velocity of the ground slide at the end of the barrel for this set up. The pellet is broken up into separated micro-particles which pass through a mesh. As expected, the velocities of the separated micro-particles decrease due to energy loss from the impaction and passage though the mesh.

111 In this paper, the mathematical formulation and it experimental validation are presented. The velocity of 112 the micro-particles is simulated as it is one of the most important variables that determine penetration 113 depth of the micro-particles in the deceleration stage. Furthermore, the model is formulated to mimic the 114 acceleration, separation and deceleration stages of MNs assisted micro-particles delivery where the 115 governing equations are solved using MATLAB (Version R2012b). It quantifies the effect of operating 116 pressure on the velocity of the ground slide and compares the results with previous experimental data 117 obtained by Zhang et al. (2013b) to verify the acceleration stage of the model. In addition, the trajectories 
of the micro-particles in the deceleration stage are simulated to determine the routes of the micro-particles

119 and distribution of the micro-particles in the three layers of skin. The developed model is used to study the

120 penetration depth of micro-particles in relation to operating pressure, particle size and MN length and

121 these are compared with a selection of experimental results for the experimental validation. Please note

122 that the paper is focused on modelling the micro-particle delivery process. The issues related to the

123 loading of genes on these particles and subsequent gene transfection in a target cells are not discussed in

124 this paper.

125

\section{2. Methodology}

1272.1 Governing equations for micro-particles transport in various stages

128 As discussed earlier, the MNs assisted micro-particles delivery process consists of acceleration,

129 separation and deceleration stages (Zhang et al., 2013a,b). Brief operation principles of each stage and

130 the corresponding governing equations that have been used to quantify the process are presented in the

131 following sections.

132

\section{$133 \quad 2.1 .1 \quad$ Acceleration stage}

134 Acceleration stage uses a compressed gas as a driving force to accelerate the ground slide to a certain

135 velocity which is controlled by the operating pressure in the receiver (see Figure 1). A compressed gas (air 136 in this case) is released from a gas cylinder and stored in the receiver (Zhang et al., 2013b). The pressure 137 inside the receiver is detected by a sensitive pressure transducer. Before the solenoid valve of the system 138 is opened, the initial volume and pressure inside the receiver are $\mathrm{V}_{1}$ and $\mathrm{P}_{1}$. After the valve is opened, the 139 gas expands and accelerates the ground slide. The volume of air increases to $V_{2}$ and the pressure 140 decreases to $\mathrm{P}_{2}$. Assuming that the gas expands adiabatically, we can apply the Boyle's law (Webster, 141 1995) to obtain:

$$
\mathrm{P}_{1} \mathrm{~V}_{1}^{\gamma}=\mathrm{P}_{2}\left(\mathrm{~V}_{1}+\mathrm{V}_{2}\right)^{\gamma}
$$

143 Where $\gamma$ is the heat capacity ratio. This process defines gas as a fluid where $\gamma=1.4$ for diatomic gas and $144 \gamma=1.6$ for a monatomic gas.

145 In the acceleration stage, only air does work on the ground slide. The work done by the gas is:

$$
\int_{0}^{\mathrm{L}} \mathrm{P}_{2} \pi \mathrm{R}^{2} \mathrm{dl}=\mathrm{E}
$$

147 Where $L$ is the length of the acceleration stage and $R$ is the radius of the ground slide. 
We define the final velocity of ground slide before reaching the stopping wall as $u$, and the mass of ground slide with the pellet as $\mathrm{M}$. The kinetic energy of the ground slide is therefore given as:

$$
\mathrm{E}=\frac{1}{2} \mathrm{Mu}^{2}
$$

152 The sliding friction of the ground slide travelling in the channel is neglected in this formulation. Based on

153 the law of conservation of energy, the velocity of the ground slide is given as:

$$
\mathrm{u}=\sqrt{\frac{2 \mathrm{P}_{1} \mathrm{~V}_{1}^{\gamma}\left[\left(\mathrm{V}_{1}+\pi \mathrm{R}^{2} \mathrm{~L}\right)^{1-\gamma}-\left(\mathrm{V}_{1}\right)^{1-\gamma}\right]}{\mathrm{M}(1-\gamma)}}
$$

\subsubsection{Separation stage}

157 In the separation stage, the compressed air is released from the vent hole (see Figure 1) and the ground slide blocks the gas from flowing into the deceleration stage (Zhang et al., 2013a,b). Further, the pellet is broken up and separated into individual micro-particles by a mesh which then move across the mesh into the deceleration stage. In this process, the initial velocity of the pellet is equal to $u$ which is the velocity of the ground slide. The pellet may lose some energy due to its impact on the mesh which separates it into individual micro-particles. Assuming that the energy loss is $x$ in this process, the process is described based on the law of conservation of energy and given as:

$$
\frac{1}{2} \mathrm{nmu}_{1}^{2}=(1-\mathrm{x}) \times \frac{1}{2} \mathrm{~m}_{\mathrm{p}} \mathrm{u}^{2}
$$

Where $\mathrm{n}$ is the number of micro-particles in the pellet; $\mathrm{m}$ is the mass of a single micro-particle; $\mathrm{u}_{1}$ is the velocity of micro-particles after passing through the stopping screen and $m_{p}$ is the mass of the pellet which can described as $\mathrm{m}_{\mathrm{p}}=\mathrm{nm}$

The rearranged equation (5) gives:

$$
\sqrt{1-\mathrm{x}} \times \mathrm{u}=\mathrm{u}_{1}
$$

171

\section{$172 \quad$ 2.1.3 Deceleration stage}

173 The deceleration stage can be separated into two parts. In the first part, the particle travels between the 174 mesh and the target. There is a gap between the mesh and target which allows spraying of the 175 micro-particles on a large-area of the target tissue. Thus, the air drag force acting on the separated 176 micro-particles should be considered in this process. The second step involves modelling the process of 177 the particle penetration in the skin, which requires consideration of the resistance force from the skin on

178 the micro-particles delivery. The micro-particles need to breach the SC and pierce the subsequent layers, 
e.g., the epidermis layer. The detailed mathematical principles of these two steps are explained in sections 2.1.3.1 and 2.1.3.2.

182 The trajectories of the micro-particles in the deceleration stage are simulated in two dimensions (2D). The initial positions and moving directions of high-speed micro-particles are randomly chosen from the beginning in the first step of the deceleration stage. The motion is considered to be linear but varying in velocity of the particles due to the effects of drag force on the particles. The particles are allowed to impact on the boundary of the gap between the mesh and skin. We define that these impacts are elastic collisions and the particles rebound on the boundary in the model. The detailed physical-mathematical principle of the micro-particle trajectories are discussed in section 2.1.3.3.

\subsubsection{Micro-particles travel in the gap between mesh and skin}

191 The separated particles decelerate in air before hitting the target (Zhang et al., 2013a,b). This process is described by the following energy balance equation:

$$
\mathrm{E}_{2}+\mathrm{E}_{\mathrm{sfe}}+\mathrm{E}_{\mathrm{d}}=\frac{1}{2} \mathrm{mu}_{1}^{2}
$$

194 Where $E_{2}$ is the final kinetic energy of the separated micro-particle. $E_{\text {sfe }}$ is the surface free energy of the pellet and $E_{d}$ is the energy lost due to the frictional drag force from the micro-particles in the air.

197 The frictional drag force on a micro-particle in the air is given as:

$$
\mathrm{F}_{\mathrm{d}}=\frac{1}{2} \rho \mathrm{u}_{2}^{2} \mathrm{C}_{\mathrm{d}} \mathrm{A}_{\mathrm{p}}
$$

Where $F_{d}$ is the force of drag, $\rho$ is the density of air, $A_{p}$ is the projected cross-sectional area of the separated micro-particle, $\mathrm{C}_{\mathrm{d}}$ is the air drag coefficient and $\mathrm{u}_{2}$ is the velocity of the separated particle in the space between mesh and target.

202

The energy loss due to the drag force is given as:

$$
\mathrm{E}_{\mathrm{d}}=\int_{0}^{\mathrm{L}_{1}} \mathrm{~F}_{\mathrm{d}} \mathrm{dl}
$$

After differentiating the energy loss $E_{d}$ with the distance $l$ we obtain:

$$
\mathrm{F}_{\mathrm{d}}=\frac{\mathrm{dE}_{\mathrm{d}}}{\mathrm{dl}}
$$

207 Where I is the gap between the mesh and target. 
The final kinetic energy of the separated particles before they hit the target is given as:

$$
\mathrm{E}_{2}=\frac{1}{2} \mathrm{mu}_{2}^{2}
$$

211 Based on equations (8) and (10), the relationship between the particle velocity and travel distance is given 212 as

$$
-\mathrm{mu}_{2} \frac{\mathrm{du}_{2}}{\mathrm{dl}}=\frac{1}{2} \rho \mathrm{u}_{2}^{2} \mathrm{C}_{\mathrm{d}} \mathrm{A}_{\mathrm{p}}=\mathrm{F}_{\mathrm{d}}
$$

214 The drag coefficient $C_{d}$ in equation (12) is an important parameter in the modelling of gas and particle 215 interactions. This coefficient is a function of the particle Reynolds number (Re), which depends on the gas viscosity $(\mu)$ and density $(\rho)$, particle diameter $(d)$, and relative velocity $\left(\mathrm{u}_{2}\right)$. For the purpose of this paper, the Reynolds number is defined as:

$$
\operatorname{Re}=\frac{\rho \mathrm{u}_{2} \mathrm{~d}}{\mu}
$$

219 The relationship between $C_{d}$ and Reynolds number, as used in this work, are shown in the appendix.

\subsubsection{Micro-particles penetration in the target tissue}

222 The particle deceleration in the target skin has the same principle with the particle travel in the air. In the 223 deceleration stage, the separated micro-particles are resisted by the tissue and their velocities are slowed down. Based on the law of conservation of energy, the drag force can be expressed as:

$$
\mathrm{f}_{\mathrm{d}}=-\mathrm{m} \frac{\mathrm{du}_{\mathrm{d}}}{\mathrm{dt}}
$$

226 Where $f_{d}$ is the drag force for the micro-particles, $u_{d}$ is the velocity of separated micro-particles in the 227 tissue.

228 For the penetration in the target, various studies have adopted that the resistant force on the micro-particle is separated into three components, namely, yield force $\left(F_{y}\right)$, frictional resistive force $\left(F_{f}\right)$ and resistive inertial force of target material $\left(F_{i}\right)$ (Soliman et al., 2011; Liu, 2007; Mitchell et al., 2003; Kendall et al. 2001; Dehn, 1987). In consistent with these previous studies, we adopt the following the force balance equation:

$$
\mathrm{m} \frac{\mathrm{du}_{\mathrm{d}}}{\mathrm{dt}}=-\left(\mathrm{F}_{\mathrm{i}}+\mathrm{F}_{\mathrm{f}}+\mathrm{F}_{\mathrm{y}}\right)
$$

234 The equation for each component of the resistant force is as shown below:

$$
\mathrm{F}_{\mathrm{i}}=6 \pi \mu_{\mathrm{t}} \mathrm{r}_{\mathrm{p}} \mathrm{u}_{\mathrm{d}}
$$




$$
\mathrm{F}_{\mathrm{f}}=\frac{1}{2} \rho_{\mathrm{t}} \mathrm{A}_{\mathrm{p}} \mathrm{u}_{\mathrm{d}}^{2}
$$

$$
\mathrm{F}_{\mathrm{y}}=3 \mathrm{~A}_{\mathrm{p}} \sigma_{\mathrm{y}}
$$

238 Where $\mu_{\mathrm{t}}$ is the viscosity of the target, $\mathrm{r}_{\mathrm{p}}$ is the radius of the micro-particle, $\rho_{\mathrm{t}}$ is the density of the target

239 and $\sigma_{\mathrm{y}}$ is the yield stress of the target.

241 2.1.3.3 The mathematical-physical principle and determination of micro-particle trajectory in

\section{2 deceleration stage}

243 To determine the trajectory of the micro-particles, the mathematical statement is formulated in two 244 dimensions. Depending on the velocity, size of particle, etc., other important features of this is the 245 possibility of different angles and positions of particle entrance which have direct implication on the 246 velocity of the micro-particles. The air in the deceleration stage might move forward when the high pressure gas pushes the ground slide forward. Although the ground slide can stop the gas from entering the deceleration stage, the air flow still happens in the front of the ground slide and causes pressure drop in the deceleration stage. Hence, the axial gas pressure drop is greater than that in the radial direction, i.e.

$$
\frac{\mathrm{dP}_{\mathrm{z}}}{\mathrm{dz}}>>\frac{\mathrm{dP}_{\mathrm{r}}}{\mathrm{dr}}
$$

$251 \mathrm{As} \frac{\mathrm{dP}_{\mathrm{z}}}{\mathrm{dz}}>>\frac{\mathrm{dP}_{\mathrm{r}}}{\mathrm{dr}}$, the axial ( $\mathrm{z}$ ) velocity component is greater than the radial $(r)$ component. Further, the 252 velocity of the separated particle in the space between mesh and target is $u_{2}$, and the initial velocity is 253 equal to $u_{1}$.

254 Therefore

$$
u_{2}^{2}=u_{z}^{2}+u_{r}^{2}
$$

255 We define that the radial velocity component as $\mathrm{k}$ percentage of the axial component.

256 Therefore

$$
\mathrm{u}_{\mathrm{r}}=\mathrm{ku}_{\mathrm{z}}
$$

In our case, $\mathrm{k}$ is defined to be 0.2 . The air in the holes of microneedles may flow when the high pressure gas pushes the ground slide along in the system (i.e., the barrel). Although, the ground slide can stop the gas from entering the deceleration stage, the air flow still happens in front of the ground slide and causes changes in pressure drop in the deceleration stage. In this case, the axial velocity component is greater than the radial component and, therefore, the $\mathrm{k}$ value should be low so that it practically imposes the above condition in the simulations. In reality, it is not easy to identify or directly measure the $\mathrm{k}$ values.

264 Therefore, we have adopted an indirect approach to ascertain the value in our case. For this purpose, we have chosen three different $\mathrm{k}$ values, i.e., $0.1,0.2$ and 0.3 , and determined the ground slide velocity for 
each case. As we have the experimental data for the velocity as well, we compared the experimentally determined data to the simulated results. The results show that at these low values $\mathrm{k}$, the ground slide velocity is not significantly affected by $\mathrm{k}$ values. More importantly, the results for the $\mathrm{k}$ value of 0.2 seem to match slightly better with the experimental results as compared to the other $\mathrm{k}$ values. On these bases, we have chosen a $\mathrm{k}$ value of 0.2 for all our simulations in this work.

\section{2.1.3.4. Micro-particle travel between mesh and target}

273 In the first part of the deceleration stage, the air drag force is presented in equation (8). According to Newton's second law:

$$
\mathrm{F}_{\mathrm{d}}=\mathrm{ma}=\mathrm{m} \frac{\mathrm{du}_{2}}{\mathrm{dt}}
$$

276 Substitution of equation (8) into equation (21) provides:

$$
\mathrm{m} \frac{\mathrm{du}_{2}}{\mathrm{dt}}=\frac{1}{2} \rho \mathrm{u}_{\mathrm{d}}^{2} \mathrm{C}_{\mathrm{d}} \mathrm{A}_{\mathrm{p}}
$$

278 For axial component, we have

$$
\mathrm{m} \frac{\mathrm{du}}{\mathrm{dt}}=\frac{1}{2} \mathrm{C}_{\mathrm{d}} \rho \mathrm{u}_{\mathrm{d}} \mathrm{u}_{\mathrm{x}} \mathrm{A}_{\mathrm{p}}
$$

For radial component

$$
\mathrm{m} \frac{\mathrm{du}}{\mathrm{dt}}=\frac{1}{2} \mathrm{C}_{\mathrm{d}} \rho \mathrm{u}_{\mathrm{d}} \mathrm{u}_{\mathrm{y}} \mathrm{A}_{\mathrm{p}}
$$

At the same time, the radial displacement component is:

$$
\frac{\mathrm{dl}_{\mathrm{r}}}{\mathrm{dt}}=\mathrm{u}_{\mathrm{r}}
$$

284 The axial displacement component is:

285

$$
\frac{\mathrm{dl}_{\mathrm{z}}}{\mathrm{dt}}=\mathrm{u}_{\mathrm{z}}
$$

286 The program uses an if statement to define the axial displacement component of the micro-particle is 287 smaller than the space between the mesh and target or the radial displacement component is smaller than $l_{y}$, the particle will impact on the walls before entering the skin.

\subsubsection{Micro-particle penetration in skin}

291 Once the micro-particles reach the surface of the target tissue, an increased resistance from the target prevents those micro-particles to move forward. The initial velocities of the micro-particles in axial and 
velocity change of the micro-particles is calculated based on the Newton second law and it is shown in equation 27 which is obtained from equation 15 :

$$
\mathrm{m} \frac{\mathrm{du}}{\mathrm{dt}}=\frac{1}{2} \rho \mathrm{u}_{\mathrm{d}}^{2} \mathrm{~A}_{\mathrm{p}}+3 \mathrm{~A}_{\mathrm{p}} \sigma_{\mathrm{y}}+6 \pi \mu_{\mathrm{t}} \mathrm{r}_{\mathrm{p}} \mathrm{u}_{\mathrm{d}}
$$

297 For the axial component of equation (27), we have

$$
m \frac{d u_{r}}{d t}=\frac{1}{2} \rho u_{d} u_{r} A_{p}+3 A_{p} \sigma_{y} \frac{u_{r}}{u_{d}}+6 \pi \mu_{t} r_{p} u_{r}
$$

On the other hand, the radial component of equation (27) is

$$
m \frac{d u_{z}}{d t}=\frac{1}{2} C_{d} \rho u_{d} u_{y} A_{p}+3 A_{p} \sigma_{y} \frac{u_{z}}{u_{d}}+6 \pi \mu_{t} r_{p} u_{z}
$$

301 In the program, we have used an if statement to define the location of micro-particle. If the axial 302 displacement component of a micro-particle is larger than the space between the mesh and target, and

303 the radial displacement component is located at a space between two holes, the particle will pierce into the target skin. If the axial displacement component of the micro-particle is larger than the space between the mesh and target and the radial displacement component is located at a hole area created by the MN, the particle is defined to be delivered in a hole. Thus, a micro-particle is defined to travel forward in the hole, penetrate into the skin, and achieve a further penetration depth inside skin. The model behaviour is explained in the section 2.3 in detail.

309

2.1.3.6. Quantitative description of the events involving micro-particle rebound between mesh and

311 target

312 In the first step of deceleration stage, the micro-particles may impact on the boundary of the gap between 313 the mesh and target skin. We define that the particles impact on the boundary of the gap between the 314 mesh and skin is an elastic collision, see Figure 2.

316 The coordinates of radial and axial velocity components at a point on $(x, y)$ axes are defined as $\left(V_{x}, V_{y}\right)$. 317 From Figure 2, it can be seen that in order to change from $(x, y)$ axes to $(r, z)$ axes, a rotation through $318\left(\frac{\pi}{2}+\theta\right)$ is required. The new coordinates of the tangential and normal velocity components of that point are $\left(V_{r}, V_{z}\right)$ on $(r, z)$ axes. Using the standard matrix rules (Eberly, 2002) to perform the axes- rotation of 320 the velocity vectors gives 


$$
\left(\begin{array}{c}
\mathrm{V}_{\mathrm{r}} \\
\mathrm{V}_{\mathrm{z}}
\end{array}\right)=\left(\begin{array}{cc}
\cos \left(\frac{\pi}{2}+\theta\right) & -\sin \left(\frac{\pi}{2}+\theta\right) \\
\sin \left(\frac{\pi}{2}+\theta\right) & \cos \left(\frac{\pi}{2}+\theta\right)
\end{array}\right)\left(\begin{array}{l}
\mathrm{V}_{\mathrm{x}} \\
\mathrm{V}_{\mathrm{y}}
\end{array}\right)=\left(\begin{array}{cc}
-\sin \theta & -\cos \theta \\
\cos \theta & -\sin \theta
\end{array}\right)\left(\begin{array}{c}
\mathrm{V}_{\mathrm{x}} \\
\mathrm{V}_{\mathrm{y}}
\end{array}\right)
$$

322 When inverted, equation (30) gives

$$
\left(\begin{array}{c}
\mathrm{V}_{\mathrm{x}} \\
\mathrm{V}_{\mathrm{y}}
\end{array}\right)=\left(\begin{array}{cc}
-\sin \theta & \cos \theta \\
-\cos \theta & -\sin \theta
\end{array}\right)\left(\begin{array}{c}
\mathrm{V}_{\mathrm{r}} \\
\mathrm{V}_{\mathrm{z}}
\end{array}\right)
$$

324

325 Equation (31) therefore suggests that for the velocity on impact, the tangential and normal velocity 326 components on the impact plane are

$$
\begin{aligned}
& \mathrm{V}_{\mathrm{xi}}=-\mathrm{V}_{\mathrm{ri}} \sin \theta+\mathrm{V}_{\mathrm{zi}} \cos \theta \\
& \mathrm{V}_{\mathrm{yi}}=-\mathrm{V}_{\mathrm{ri}} \cos \theta-\mathrm{V}_{\mathrm{zi}} \sin \theta
\end{aligned}
$$

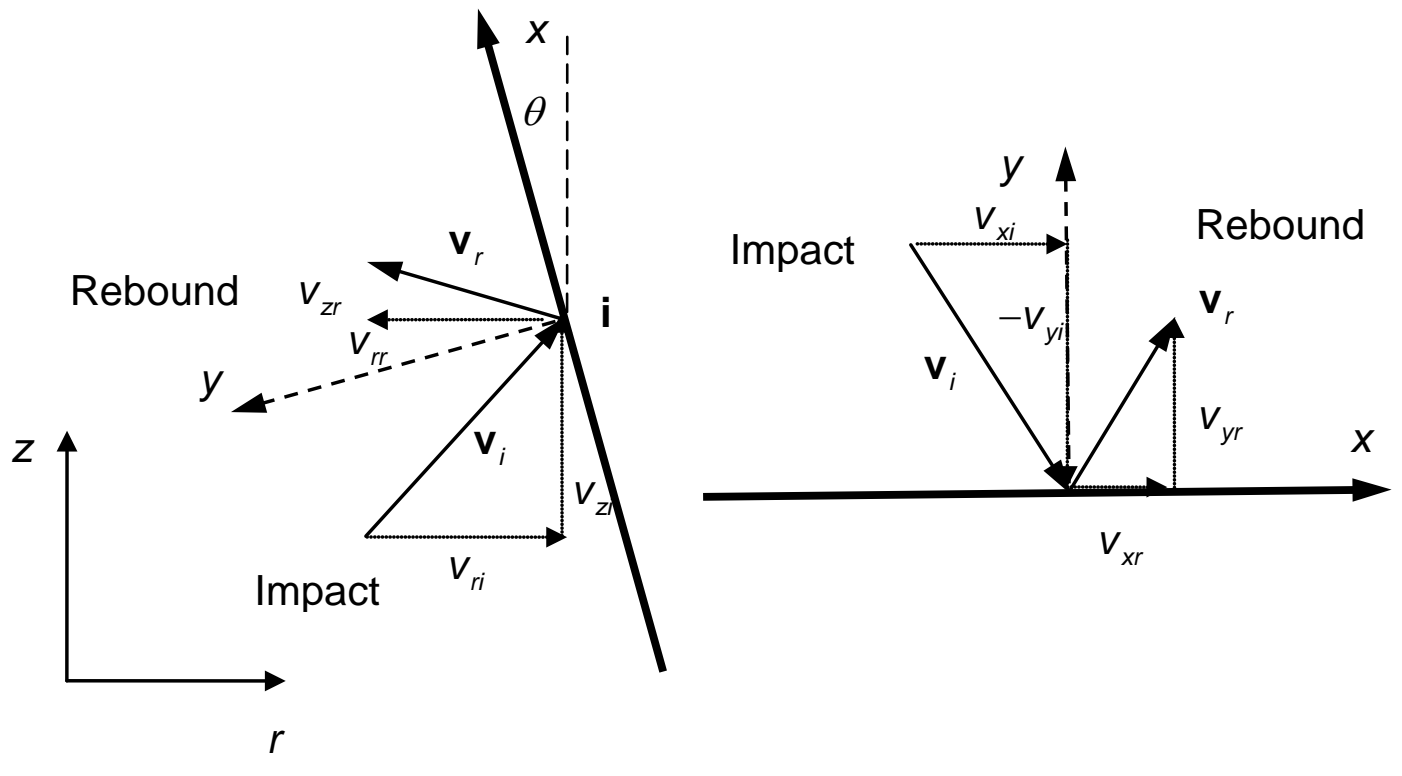

328

Figure 2: The trajectory of particle impact on a planar wall

331 For the velocity on rebound, the tangential and normal velocity components may be written in terms of the

332 following coefficients of restitution

$$
\begin{gathered}
\mathrm{V}_{\mathrm{xr}}=\mathrm{e}_{\mathrm{t}} \mathrm{V}_{\mathrm{xi}} \\
\mathrm{V}_{\mathrm{yr}}=-\mathrm{e}_{\mathrm{n}} \mathrm{V}_{\mathrm{yi}}
\end{gathered}
$$


334 Hence, rotating back through $\left(\frac{\pi}{2}+\theta\right)$, using equation (30), to get the radial and axial velocity components on rebound provide:

$$
\begin{aligned}
& \mathrm{V}_{\mathrm{rr}}=-\mathrm{e}_{\mathrm{t}} \sin \theta\left(-\mathrm{V}_{\mathrm{ri}} \sin \theta+\mathrm{V}_{\mathrm{zi}} \cos \theta\right)+\mathrm{e}_{\mathrm{n}} \cos \theta\left(-\mathrm{V}_{\mathrm{ri}} \cos \theta-\mathrm{V}_{\mathrm{zi}} \sin \theta\right) \\
& \mathrm{V}_{\mathrm{zr}}=\mathrm{e}_{\mathrm{t}} \cos \theta\left(-\mathrm{V}_{\mathrm{ri}} \sin \theta+\mathrm{V}_{\mathrm{zi}} \cos \theta\right)+\mathrm{e}_{\mathrm{n}} \sin \theta\left(-\mathrm{V}_{\mathrm{ri}} \cos \theta-\mathrm{V}_{\mathrm{zi}} \sin \theta\right)
\end{aligned}
$$

We define the collisions between micro-particles and the boundary of the gap between mesh and skin are elastic collision, and then the coefficient of restitution is equal to 1 . It means that there is no energy lost due to the rebound; only the direction of motion has been changed.

341 Table 1: The meaning of each variable in Figure 2

\begin{tabular}{|c|l|}
\hline Variables & \multicolumn{1}{|c|}{ Description } \\
\hline$V_{\mathrm{i}}$ & The velocity on impact \\
\hline$V_{\mathrm{ri}}$ & The radial velocity component on impact \\
\hline$V_{\mathrm{zi}}$ & The axial velocity component on impact \\
\hline$V_{\mathrm{r}}$ & The velocity on rebound \\
\hline$V_{\mathrm{rr}}$ & The radial velocity component on rebound \\
\hline$V_{\mathrm{zr}}$ & The axial velocity component on rebound \\
\hline$\Theta$ & The angle of boundary of the gap between mesh and skin \\
\hline $\mathrm{I}$ & The impact point \\
\hline$V_{\mathrm{xi}}$ & The tangential velocity component on impact \\
\hline$V_{\mathrm{yi}}$ & The normal velocity component on impact \\
\hline$V_{\mathrm{xr}}$ & The tangential velocity on rebound \\
\hline$V_{\mathrm{yr}}$ & The normal velocity on rebound \\
\hline
\end{tabular}

\section{$343 \quad 2.2$ Selection of modelling parameters}

344 The impact velocity, particle size and density, target properties are defined as the major variables affecting

345 the penetration depth. The layers of the skin are considered to mimic the human skin in the model (see

346 Figure 3a). The skin is divided into two distinct macroscopic layers known as the dermis and the epidermis 347 (Parker, 1991; Phipps, 1988). Stratum corneum is considered as a part of the epidermis layer. Therefore, 348 the skin is considered to have three layers in the model, which are shown in Figure $3 \mathrm{~b}$ which is a 349 magnified profile of a section of the model geometery shown in Figure 3a. The thicknesses of the different 
skin layers differ which are listed in Table 2. The viscosity of each layer is treated as the same in the model.

351 Previously, Zhang et al. (2014) have analysed the viscosity of the porcine skin using a rotational 352 viscometer, which will be used as a replacement for human skin in the model and shown in Table 4. For 353 example, the density of the stratum corneum and viable epidermis are defined as 1.5 and $1.15 \mathrm{~g} / \mathrm{cm}^{3}$, respectively, in consistent with the results of Duck (1990). Wildnauer et al. (1971) have shown that the yield stress of stratum corneum range from 3.2 to $22,5 \mathrm{MPa}$, which have been obtained from the measurements of stress-strain characteristics of the human stratum corneum samples.

Table 2: Skin properties used in the model

\begin{tabular}{lll}
\hline Parameter & Value & Reference \\
\hline Thickness of $\mathrm{VE}, \mathrm{T}_{\mathrm{ve}}(\mathrm{m})$ & 0.0001 & Holbrook et al. (1974) \\
Thickness of $\mathrm{SC}, \mathrm{T}_{\mathrm{sc}}(\mathrm{m})$ & 0.00002 & Matteucci et al. (2008); \\
& & Schaefer et al. (1996) \\
Yield stress of $\mathrm{SC}, \mathrm{Y}_{\mathrm{sc}}(\mathrm{MPa})$ & $3.2-22.5$ & Wildnauer et al. (1971) \\
Density of $\mathrm{SC}, \rho_{\mathrm{sc}}\left(\mathrm{g} / \mathrm{cm}^{3}\right)$ & 1.5 & Duck (1990) \\
Density of $\mathrm{VE}, \rho_{\mathrm{ve}}\left(\mathrm{g} / \mathrm{cm}^{3}\right)$ & 1.15 & Duck (1990) \\
Yield stress of $\mathrm{VE}, \mathrm{Y}_{\mathrm{ve}}(\mathrm{MPa})$ & 2.2 & Kishino et al. (1988) \\
Viscosity of skin, $\mu_{\mathrm{t}}(\mathrm{Pas})$ & 19.6 & Zhang et al. (2014) \\
\hline
\end{tabular}

360 In order to investigate the MN effect on the penetration depth, an in-house fabricated MN, Adminpatch MN 3611500 and 1200 are (nanoBioSciences limited liability company, Sunnyvale, CA, USA) chosen for both model and experiments. The detailed characterizations of each MN are shown in Figure 4 and explained in the Table 3. Zhang et al. (2014) have chosen Adminpatch MN 1500 to analyse the MNs assisted micro-particles delivery and show that the lengths of pierced holes vary after the removal of the MNs. But, the length of the pierced holes are considered uniform in the model and presented in Table 4. It is worth mentioning that the lengths of the pierced holes are from a study of the insertion of $\mathrm{MN}$ in a skin mimicking agarose gel $(0.0265 \mathrm{~g} / \mathrm{ml}$ of agarose), which has been obtained in a previous work (Zhang et al., 2014). McAllister et al. (2003) have assumed that after the removal of the MNs the surface area of the hole shrink to 60 percent of that the MNs which originally create the holes. In consistent with McAllister et al.(2003), the hole width is considered to be $60 \%$ of the width/radius of the MN (Table 4) at the time the

371 micro-particles are delivered. The details can be shown in Figure 3a, which presents the pierced holes as 372 uniform cones. 
374 The relevant simulation parameters of the the proposed gene gun system are obtained from an 375 experimental rig, which include the mass of the ground slide with pellet, volume of receiver, barrel length 376 and radius and space between mesh and target (skin). Spherical and irregular stainless steel particles of

37718 and $30 \mu \mathrm{m}$ average diameters are chosen to study the effects of particle size on the penetration depth 378 for both the model and experiment. The details of the relevant parameters used in the model are listed in 379 Table 4.

380

381 Table 3: The characterizations of the MN array (Zhang et al., 2013b)

\begin{tabular}{lll}
\hline Name & Parameters & Value $(\boldsymbol{\mu m})$ \\
\hline Adminpatch MN 1500 & Length & 1500 \\
& Width & 480 \\
& Thickness & 78 \\
& Space between MNs & 1546 \\
Adminpatch MN 1200 & Length & 1200 \\
& Width & 480 \\
& Thickness & 78 \\
In-house fabricated MN & Space between MNs & 1252 \\
& Length & 750 \\
& Diameter & 250 \\
& Space between MNs & 500 \\
\hline
\end{tabular}

382

383 
(a)

385

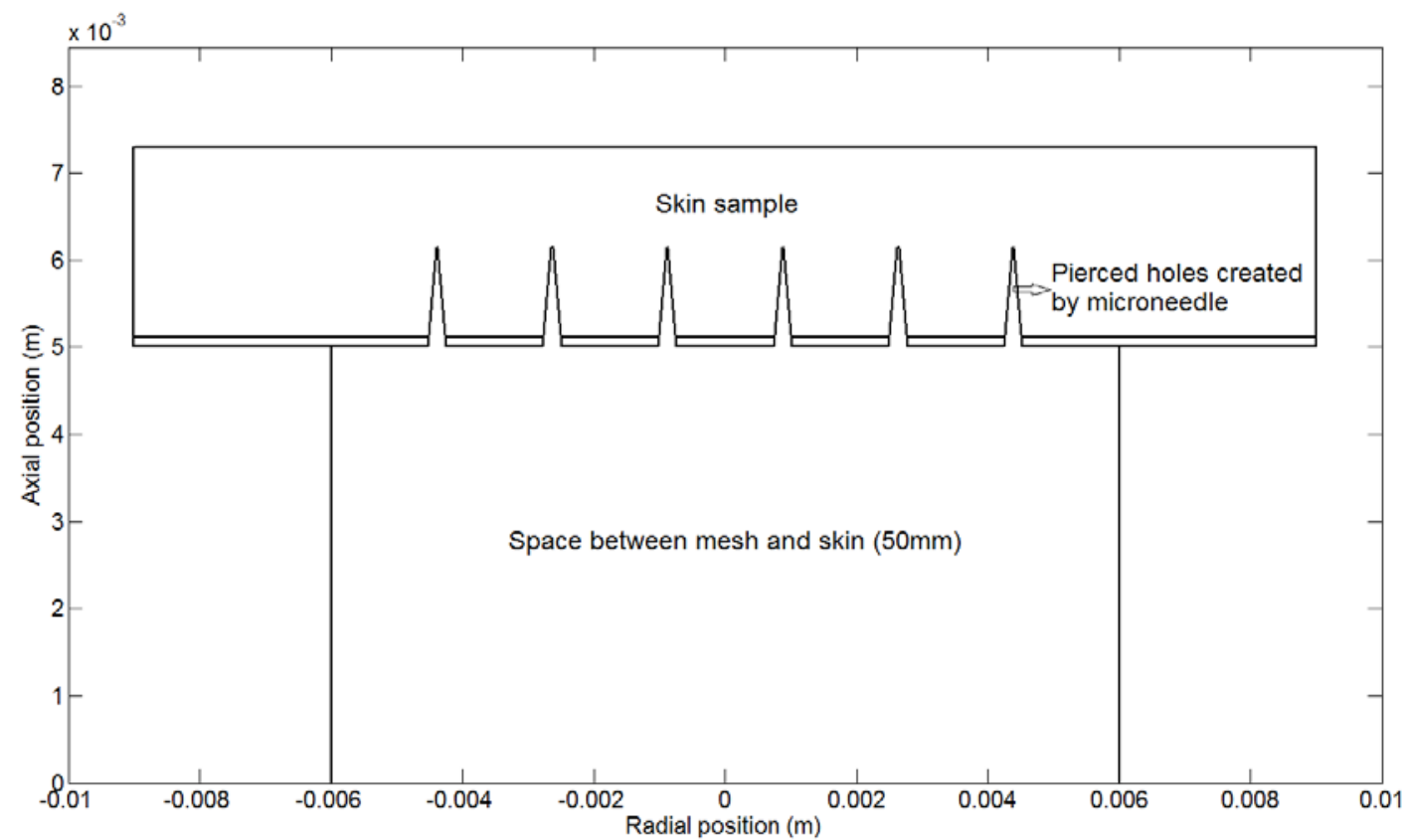

386 (b)

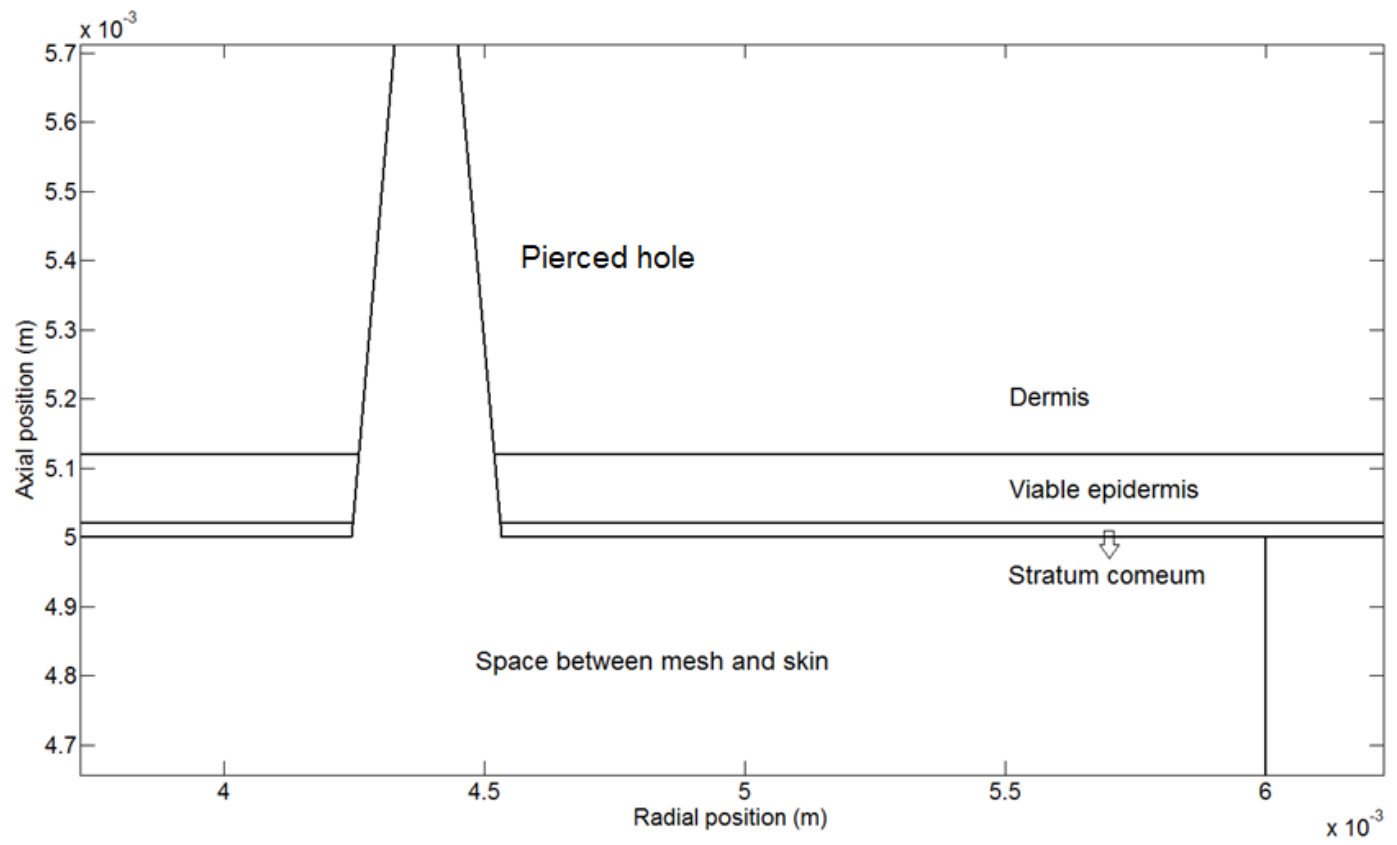

Figure 3: Structure of the deceleration stage (Adminpatch 1500): (a) the overall view of the deceleration stage, (b) the zooming view to show the skin layers 


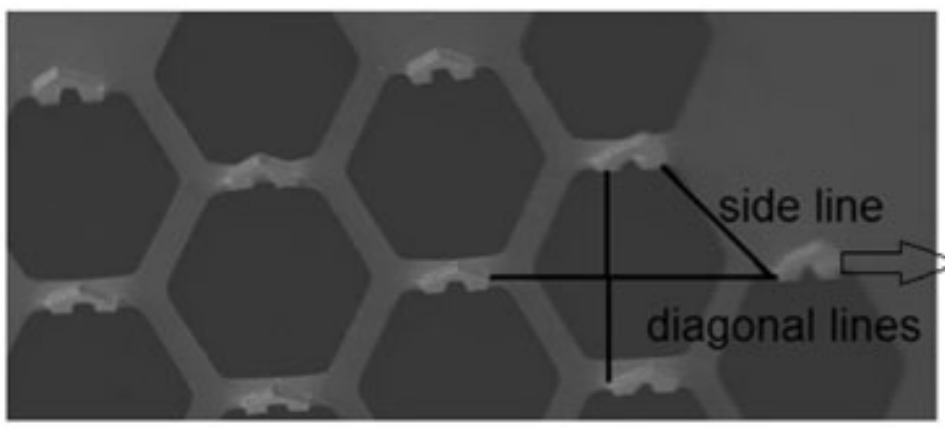

$6 \mathrm{~mm}$

(a)

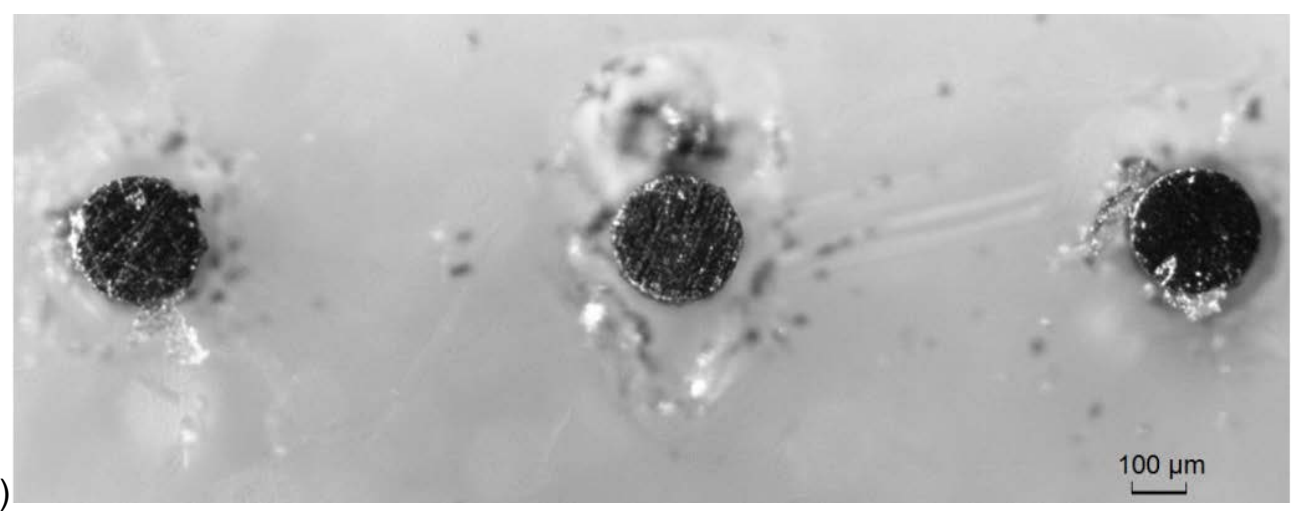

391

(b)

Figure 4: The image of MN arrays: (a) Adminpatch 1200 (b) In-house fabricated MN arrays

393

Table 4: Relevant constants used in the developed model

\begin{tabular}{|c|c|}
\hline Parameter & Value \\
\hline Mass of ground slide with the pellet, M (g) & 1.25 \\
\hline Length of barrel, $\mathrm{L}(\mathrm{m})$ & 0.5 \\
\hline Radius of barrel/ground slide, $\mathrm{R}(\mathrm{m})$ & 0.00375 \\
\hline Volume of receiver, $\mathrm{V}_{1}(\mathrm{~L})$ & 1 \\
\hline Space between mesh and skin, $\mathrm{L}_{1}(\mathrm{~m})$ & 0.05 \\
\hline Density of stainless steel $\left(\mathrm{g} / \mathrm{cm}^{3}\right)$ & 8 \\
\hline Average Diameter of spherical stainless steel particle $(\mu \mathrm{m})$ & 18 \\
\hline Average Diameter of Irregular stainless steel particle $(\mu \mathrm{m})$ & 30 \\
\hline Viscosity of air, $\mu$ (Pas) & 1.78 \\
\hline Length of pierced holes $\mathrm{L}_{\mathrm{p}}(\mu \mathrm{m})$ Adminpatch 1500 & 1149 \\
\hline Adminpatch 1200 & 1048 \\
\hline In-house fabricated MN & 656 \\
\hline Width of pierced holes $L_{w}(\mu \mathrm{m})$ Adminpatch 1500 & 302 \\
\hline Adminpatch 1200 & 302 \\
\hline In-house fabricated MN & 156 \\
\hline
\end{tabular}




\subsection{Solving governing equations}

397 The governing equations for modelling the MNs assisted micro-particles delivery are solved using 398 MATLAB (Version R2012b). MATLAB is a powerful programming software for computing and data processing and visualisation. For our case, we have used MATLAB's in-built programming language to simulate the particle delivery process in each stage. The presented model consists of a main program to explain the overall process of the micro-particles delivery, several function programs to input the integration of the required mathematical equations, event programs to define the event locators of the rebound and impact points. All constant variables (e.g. skin properties, particle properties) are included as declared global variables at the start of the program with comments in the main program for the following simulation (see Tables 2 - 4). The acceleration stage is analysed in one-dimensional using equation (4) in the main program to predict the velocity from the beginning to the end of the barrel at various pressures. The separation stage is implemented using equation (6) in the main programme to define the energy loss of micro-particles during separation stage and then to calculate the velocity of micro-particles after the passage through the mesh.

411 The presented model is focused on determining the trajectories of micro-particles in the deceleration stage.

412 Firstly, the initial velocity of separated micro-particles in the deceleration stage $\left(\mathrm{u}_{2}\right)$ is defined to be equal 413 to $\mathrm{u}_{1}$ which is the final velocity of the separated micro-particles after passing though the mesh. The 414 velocity of the separated micro-particles is then analysed in relation to time. After that a two dimensional 415 figure corresponded to the structure of the deceleration stage is prepared based on the size of 416 experimental set up as shown in Figure 3. The initial position and moving direction of high-speed 417 micro-particles are randomly chosen from the beginning in the first step of the deceleration stage to mimic 418 the condition of micro-particles passage through the mesh. The motion is considered to be linear but 419 varying in velocity of the particles due to the effect of drag force. However, the mathematical equations 420 used to determine the particle velocity are implemented in a separate function. An if statement is used to 421 determine the selection of equations to calculate the particle velocity at different positions. This program is 422 implemented to the main program by considering the condition of the function program (stiff/non-stiff) to 423 choose a suitable ode solver to determine the velocity changing of micro-particles and plot the trajectories 424 in the pre-plotted figure (Figure 3). The penetration depth of micro-particles in the skin is obtained from the 425 figure. In addition, equations $(12,15)$ are solved using a separately function program and implemented 426 into the main program to predict the penetration depth of micro-particles with/without using MNs in one-dimensional simulation. A for statement is used to repeat the same procedure to simulate a number of 
micro-particles trajectories in the program. However, the number of micro-particles is defined as a constant and inputted at the start of the main program.

430

431 Event program is used to define the impact points on the skin and the boundary of the gap between mesh 432 and skin, and further to point out the end position of micro-particles inside the skin. Setting the events 433 cause the solver to stop the integration when the micro-particles impact on the skin and the boundary of 434 the gap between mesh and skin, and then restarts the integration corresponding to the continuous moving 435 of a micro-particle. In addition, an event causes the solver to stop the integration when the velocity of 436 micro-particles is less than $10^{-10} \mathrm{~m} / \mathrm{s}$. This event program is implemented in the main program to predict 437 the events of micro-particles (e.g. impact on the boundary, penetrate into the skin) and show on the 438 particle trajectories.

439

\section{3. Results and Discussions}

\section{$441 \quad 3.1$ Acceleration stage}

442 The micro-particle velocity is a key variable in the micro-needle assisted micro-particles delivery process 443 which is simulated and discussed in this section. As mentioned earlier, the developed mathematical model 444 is built to simulate the acceleration stage of this process where a number of variables are considered such 445 as the mass of the ground slide (including the pellet), volume of the gas receiver and, barrel length and 446 diameter. The relevant constants are listed in Table 4.

448 The operating pressure is another key variable in the model which affects the velocity of the ground slide.

449 The principle of modelling the acceleration stage is explained in section 2.1.1. Indeed the model results 450 show that the operating pressure has a significant effect on the ground slide velocity (Figure 5). As can be 451 seen, an increase in the operating pressure causes an increased ground slide velocity. The velocities of 452 ground slide reach 85.5, 102.2, 125.1, 138.3 and $144.5 \mathrm{~m} / \mathrm{s}$ at $2.1,3,4.5,5.5$ and 6 bar pressures, 453 respectively. 


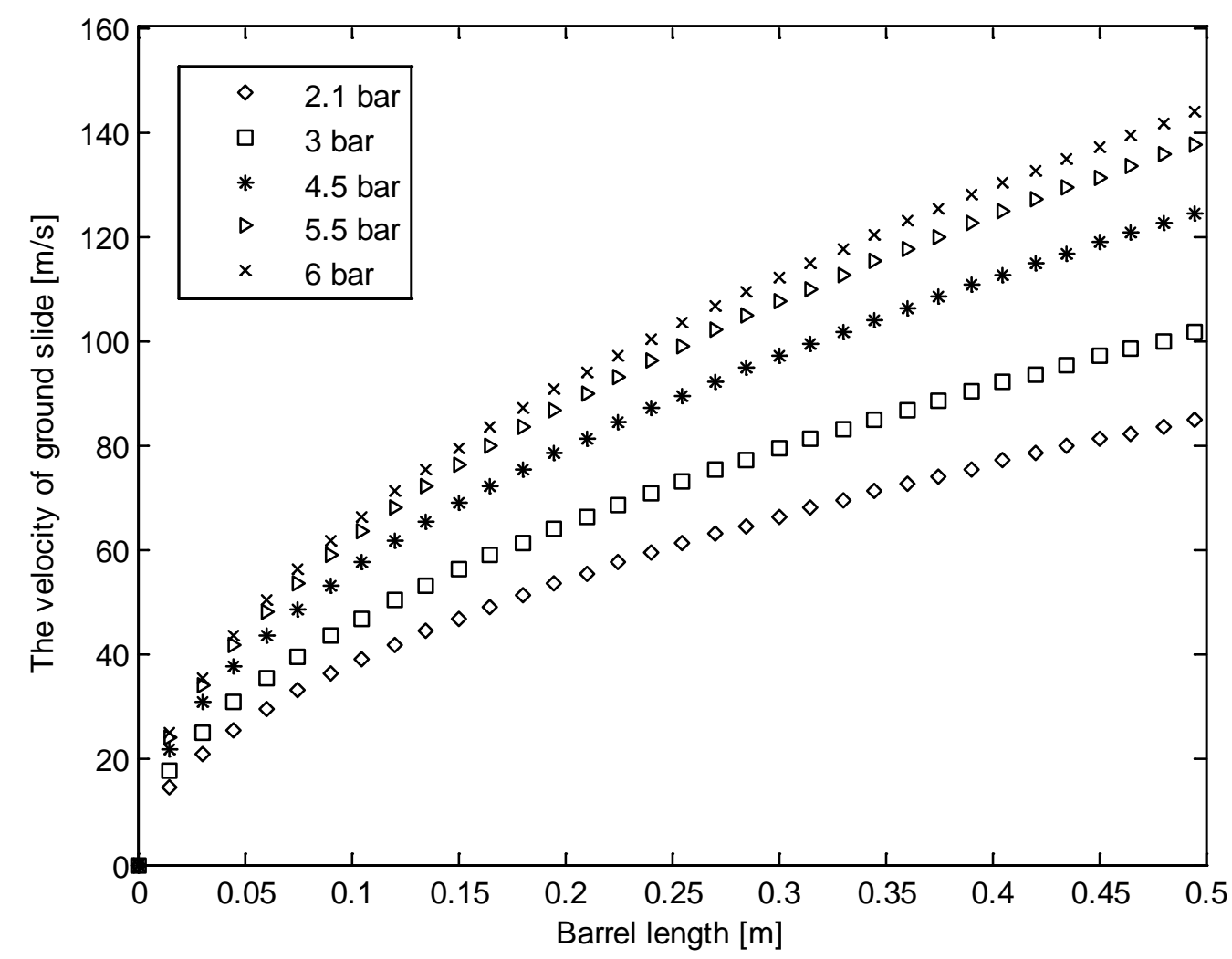

Figure 5: Effect of the operating pressure on the ground slide velocity (modelling results)

457 Zhang et al. (2013b) have used a pair of photoelectric sensors to test the velocity of the ground slide in a 458 set of experiments. A comparison is made between the results from the developed model and Zhang et al. 459 (2014)'s experiments which are shown in Figure 6. As can be seen, both sets of results compare well at 460 the chosen pressures. The velocity increases from an increase of operating pressure due to an increased 461 kinetic energy of the ground slide. This set of results provides the confidence that the developed model is 462 suitable for modelling the acceleration stage of the micro-needle assisted micro-particles delivery.

464 It is seen that the predicted velocity is comparable with the velocity of the ground slide based gene gun 465 system, e.g. light gas gun (Crozier, 1957; Mitchell at al., 2003) which can accelerate the micro-particles to velocities of $170,250,330 \mathrm{~m} / \mathrm{s}$ at 20, 40 and 60 bar pressure. In this work the ground slide is operated at 6 bar which shows that the velocity is slightly different with the LGG operated at 20 bar pressure. However, the velocity is much slower if it is compared with that of other gene gun systems, e,g. contoured shock tube (Truong et al., 2006; Mitchell et al., 2003), converging-diverging nozzle (Kendall et al., 2004a) and conical nozzle (Quinlan et al., 2001). The micro-particles normally can achieve a supersonic speed based

471 on a needle free powder injection system, such as golden particle injector which may reach a velocity over 
than $600 \mathrm{~m} / \mathrm{s}$ at 60 bar pressure using contoured shock tube (Liu et al., 2006; Mitchell et al., 2003). This is

473 because the effects of the ground slide which slows down the particle velocity.

474

475 An insufficient velocity means that the micro-particles cannot reach the desired depth inside the target due 476 to the insufficient momentum. As the operating pressure was limited in the experiments of Zhang et al. 477 (2013c), the velocity of the ground slide is simulated to reach a velocity of $457 \mathrm{~m} / \mathrm{s}$ at $6 \mathrm{MPa}$ using the 478 model, which is much higher than the velocity obtained from LGG. Zhang et al. (2013b) have shown that 479 the velocity increases with a decrease in barrel diameter and ground slide mass. Therefore, the velocity 480 can improve by changing those two objects in Zhang et al. (2013b)'s experimental set up if higher velocity 481 is necessary. Furthermore, Zhang et al. (2014) suggest that the penetration depth is maximized by using 482 MNs. The MNs make up for the insufficient velocity of the micro-particles since the pierced holes created 483 by MNs provide a positive effect on the penetration depths. This is explained more in section 3.2.

484

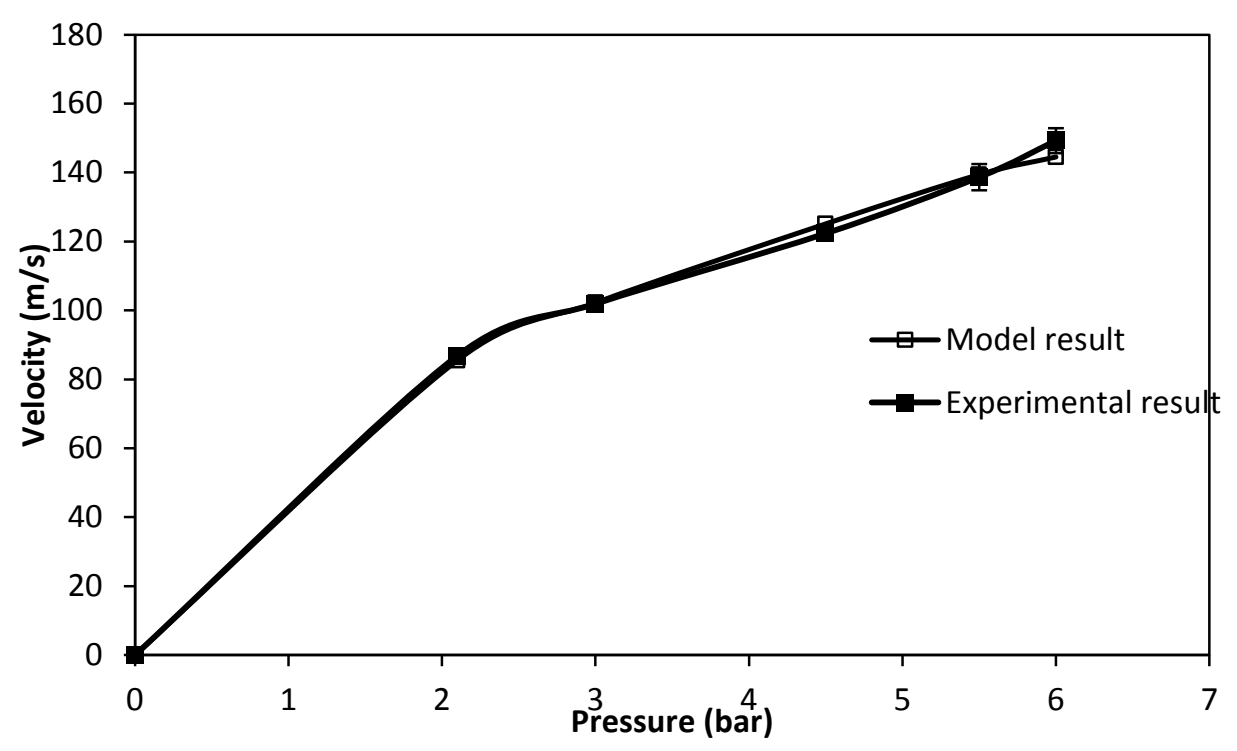

485 Figure 6: Comparison of modelling (this work) and experimental (Zhang et al., 2013b) results of the ground slide velocity against the operating pressure. The experimental results in the figure are generated from three repeats of experiments.

488

489

\subsection{Deceleration stage}

\subsubsection{The trajectory of the micro-particles}

491 Zhang et al. (2013b) have shown that a pellet can be separated into individual particles with a few agglomerates using a mesh which then can penetrate into the target. In this paper, the presented mathematical model is used to simulate the trajectories of stainless steel micro-particles of $30 \mu \mathrm{m}$ average

494 diameter in the deceleration stage. As presented in Figure $7 \mathrm{a}$, the velocity of the micro-particles is 
represented by the coloured trajectory. It is found that the velocity variation is negligible before they reach

496 the target as the effect of air drag force on the micro-particles is low. It is also found that the velocity

497 reaches approximately $131 \mathrm{~m} / \mathrm{s}$ at 5 bar operating pressure according to the results in the figure. In

498 addition, the micro-particles rebound on the boundary of the gap between mesh and skin is clearly shown

499 in Figure 7a. However, the penetrations of micro-particles in the target are not visible in this figure.

500

501 In theory, the particle velocity must decrease fast after penetrating into the skin due to an increased

502 resistance to its motion. The variation of the velocity is shown in more detail in Figure $7 \mathrm{~b}$, which is

503 obtained from zooming in a part of Figure 7a. As can be seen, the micro-particles only penetrate slightly in

504 the stratum corneum layer of skin. The detailed penetration depth refers to the resultsof dashed line in

505 Figure 8 (see the zoomed view of the axis), which shows that the stainless steel micro-particles of $30 \mu \mathrm{m}$

506 diameter only penetrate around $1.9 \mu \mathrm{m}$ inside the stratum corneum.

507

508 Figure $7 \mathrm{a}$ also shows that a number of the micro-particles achieve a further penetration depth via the 509 pierced holes. Some of them reaches the hole tip area as shown in Figure 7c. The velocity is changed only 510 slightly in the pieced holes and decrease fast after penetrating the dermis layer of the skin. The

511 penetration depth of the micro-particles via the pierced holes is shown in Figure 8 (see the zoom in view of 512 the axis). As can be seen, the penetration depth of the micro-particles reaches $1151.1 \mu \mathrm{m}$ when 513 Adminpatch MN 1500 is used. However, some particles cannot reach the holes tip and penetrate through

514 the skin surface of the pierced holes to achieve a further depth inside the epidermis/dermis layer of the 515 skin as shown in Figure $7 \mathrm{~d}$.

516

517 Finally, delivery of an arbitrarily selected number of micro-particles, namely one hundred (100) 518 micro-particles, has been simulated to determine the particle's final location in each layer of skin. As 519 presented in Figure 9, it shows that about $75 \%$ of micro-particles stop inside the stratum corneum, $2 \%$ is 520 located within the epidermis layer, and $23 \%$ penetrates further into the dermis layer. In addition, the 521 micro-particles stopped inside the epidermis or dermis layer are considered to penetrate through the 522 pierced holes, which illustrate the use of the Adminpatch MN 1500 allowing approximately 25\% of the 523 micro-particles penetration in the skin via the pierced holes. The detailed effects of the MN length, particle 524 size and operating pressure on the penetration depth are explained in the following sections. 
526

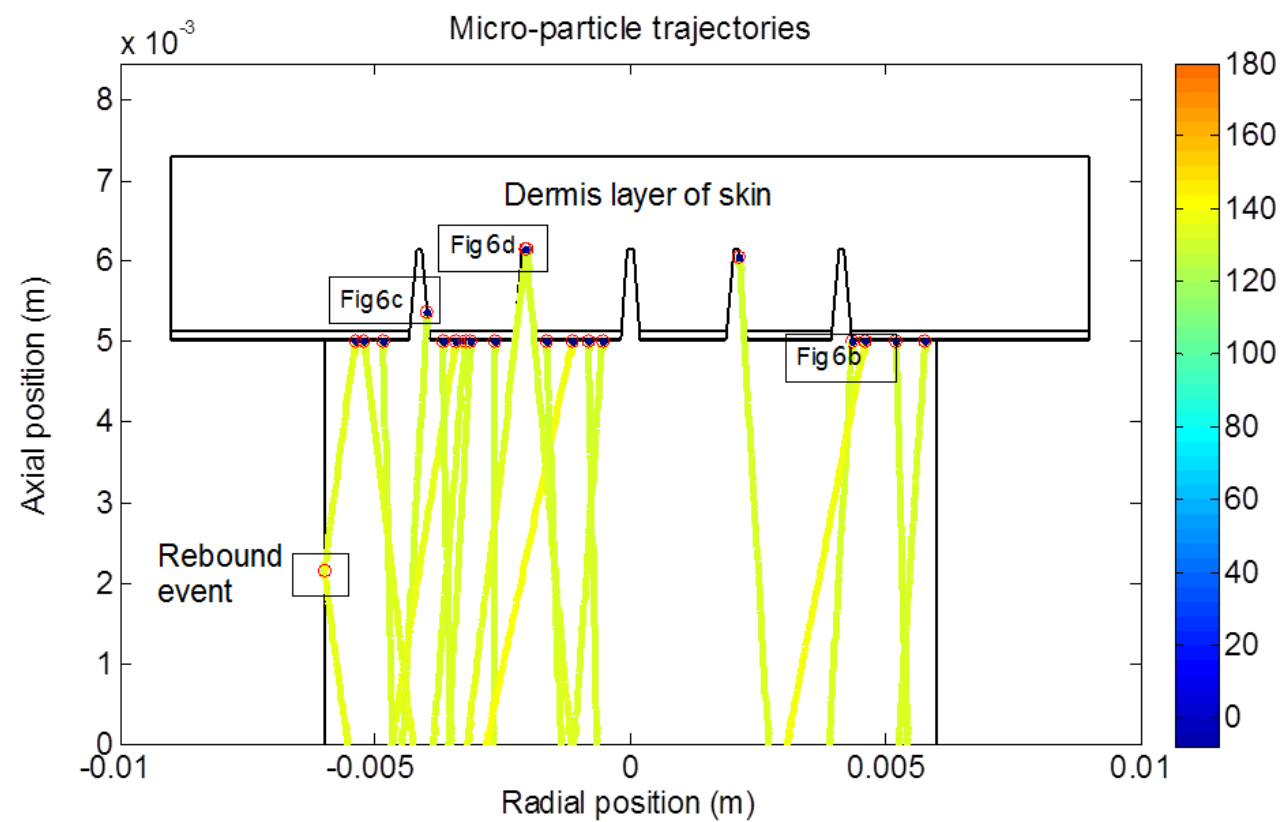

527

$528 \quad$ (a)

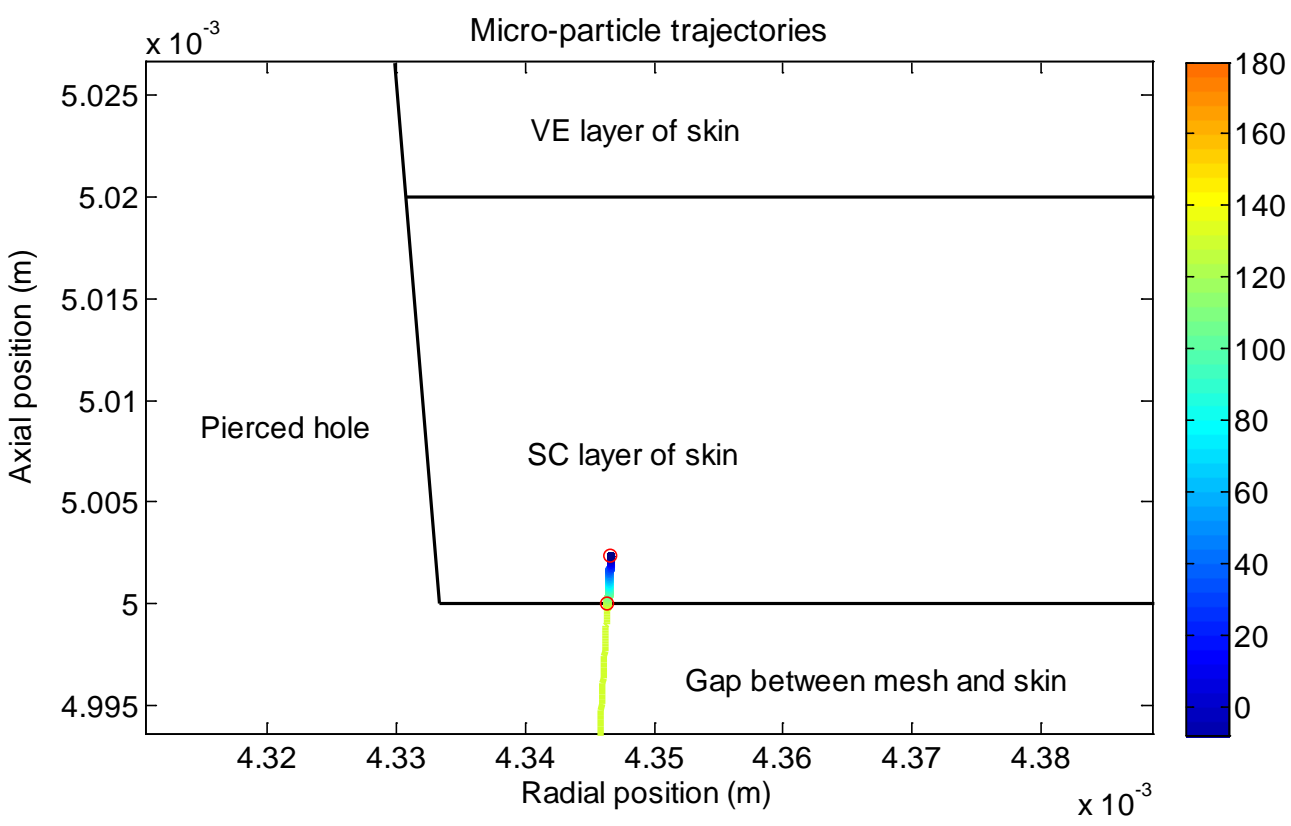

$530 \quad$ (b) 


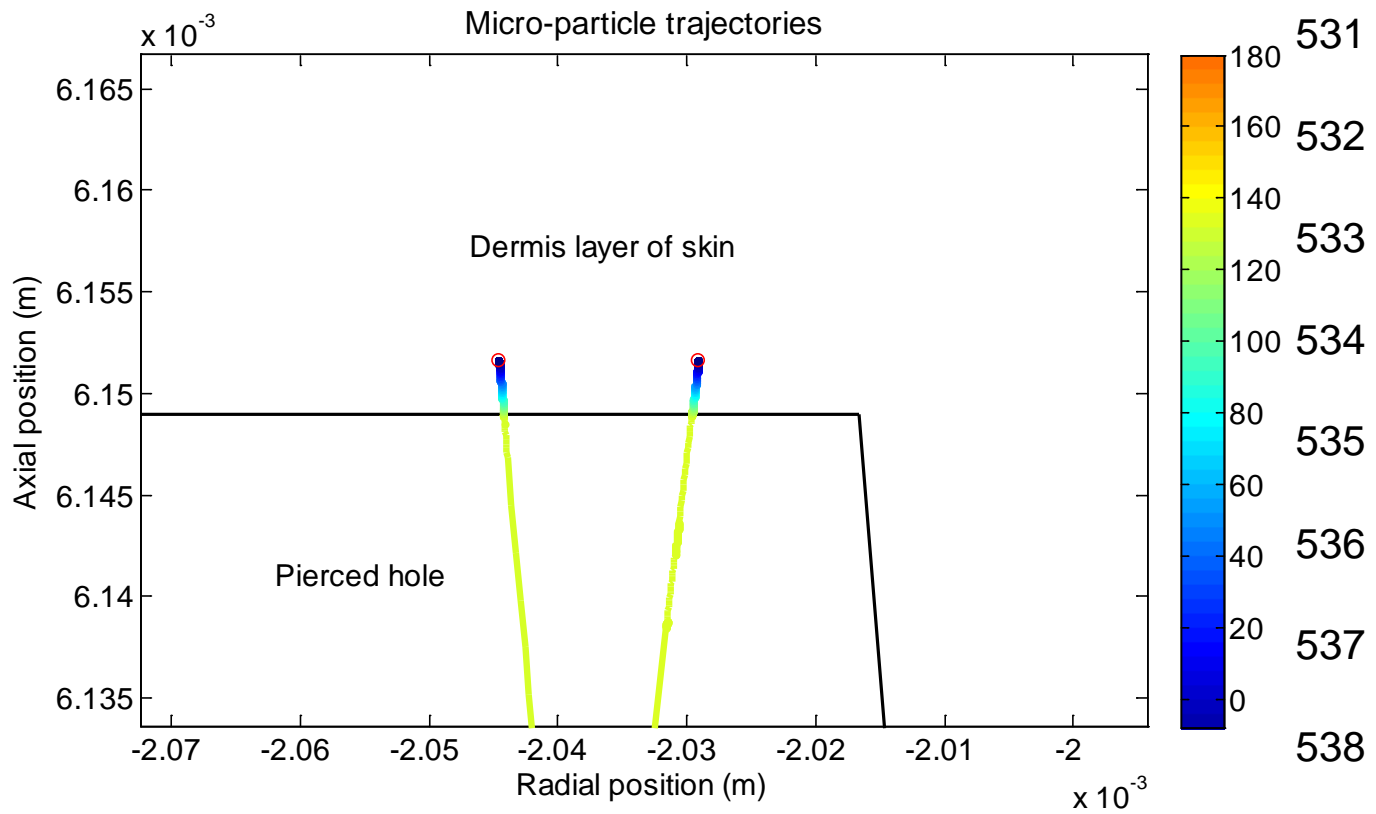

539

$540 \quad$ (c)

541

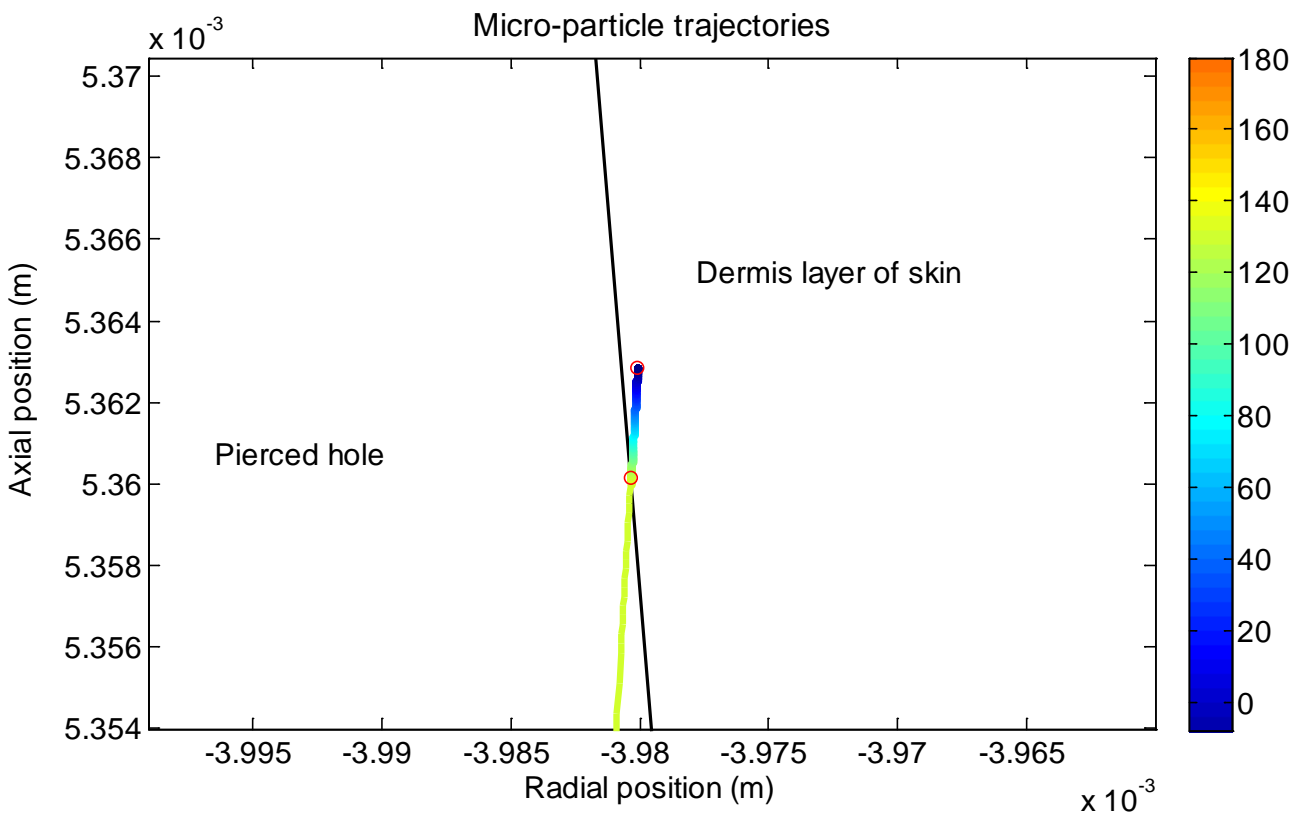

542

543

544

545

546

547

548

Radial position (m)

$\times 10$

549 (d)

550 Figure 7: The trajectories of the micro-particles in the deceleration stage for the MN assisted

551 micro-particles delivery: (a) The overall view of the micro-particles trajectories, (b) The particle penetration

552 at the area without needle hole, (c) The particle penetration at the hole tip area inside skin (d) Particle

553 penetrates into the side surface of the needle hole inside skin (stainless steel micro-particles of 30

554 diameter; pressure: 5 bar) 


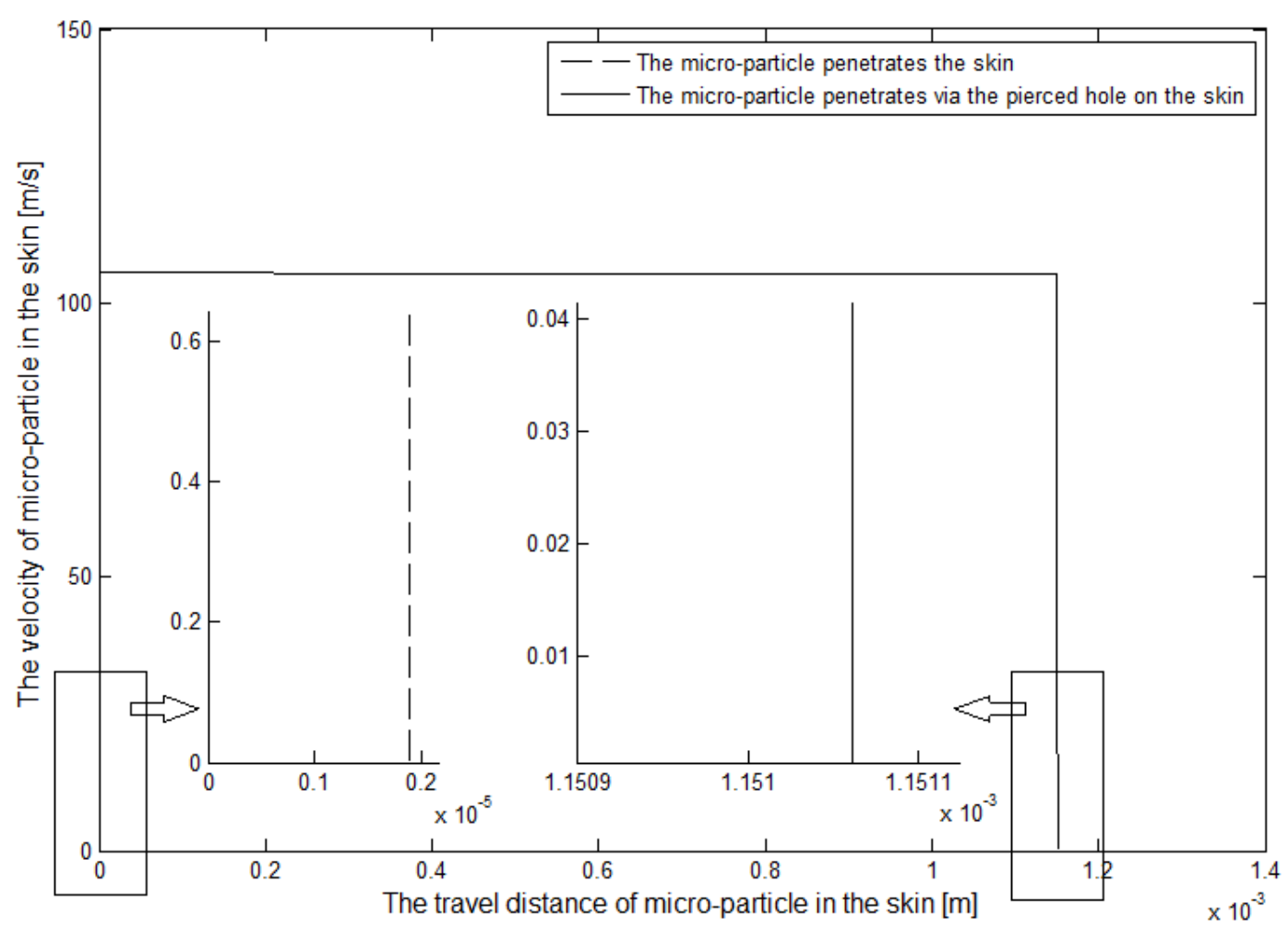

Figure 8: The travel distance of micro-particles in the skin against the velocity.

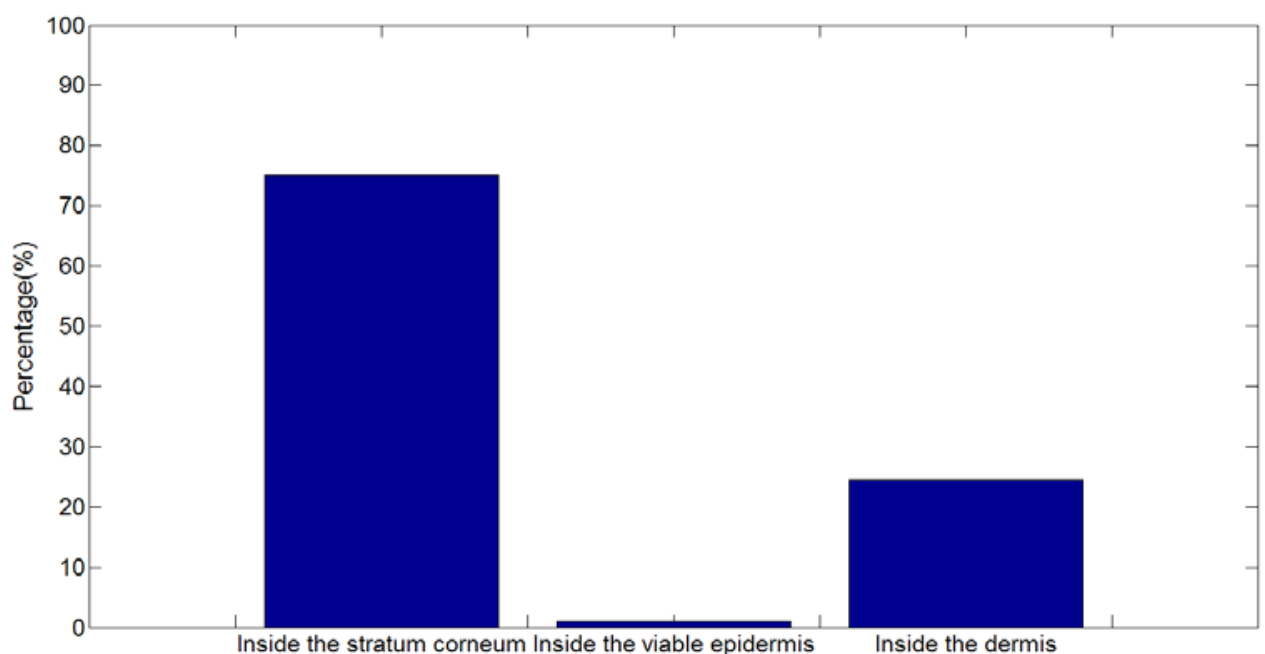

Figure 9: The distribution of the micro-particles in different layers of skin

\section{$560 \quad$ 3.2.2 Comparison with experimental results}

561 As mentioned earlier, Zhang et al. (2013c) have used an agarose gel to mimic the skin on the basis of 562 rheological properties using a rotational viscometer. The work shows that the rheology of $0.0265 \mathrm{~g} / \mathrm{ml}$ 563 concentration of agarose gel matches well with that of porcine skin, and this skin mimicking agarose gel is

564 used as a target instead of human skin to analyse the penetration depth in relation to the operating 
565 pressure, particle size and needle length. The operating pressure is varied from 3 to 5 bar to accelerate

566 biomedical grade stainless steel micro-particles of 18 and $30 \mu \mathrm{m}$ average diameters to analyse the effect

567 of the particle size and operating pressures on the penetration depths of the particle. Three different

568 lengths of micro-needle arrays, which are in-house fabricated MN $(750 \mu \mathrm{m})$ and Adminpatch MN 1500

$569(1500 \mu \mathrm{m})$ and $1200(750 \mu \mathrm{m})$ are chosen to investigate the effect of MN length on the penetration depth.

570 Results show that the penetration depth of micro-particles increases from an increase of particle size,

571 operating pressure and MN length.

572

573 In the following sections of this paper, the experimental results obtained from the previous work are 574 compared with model results to verify the applicability of the model and further understand the MNs 575 assisted micro-particles delivery.

576

\section{3.2.2.1 The effect of the operating pressure and particle size on penetration depth}

578 In general, operating pressure is one of the major variables which affect the momentum of the 579 micro-particles and is expected to affect the penetration depths of the micro-particles inside a target. To 580 confirm the significance of this effect, delivery of stainless steel micro-particles of 18 and $30 \mu \mathrm{m}$ average 581 diameters are simulated at operating pressure varies from 3 to 60 bar. The results of these simulations are 582 presented in Figure 10. In the figure, the solid lines represent the micro-particles delivery without the MN 583 pierced holes and they correspond to the primary y axis (y1). The penetration depths of the micro-particles

584 via the pierced hole are considered through the secondary y axis (y2). As can be seen, the results show 585 that an increase of the operating pressure causes a slight increase in the penetration depth. It also shows 586 that stainless steel micro-particles of $18 \mu \mathrm{m}$ diameter can penetrate only from 0.58 to $2.59 \mu \mathrm{m}$ in the skin 587 (inside the stratum corneum) in the pressure ranging from 3 to 60 bar. However, a number of 588 micro-particles delivered through the pierced holes penetrate into the dermis layer. The penetration depth 589 inside the dermis layer is slightly more than the stratum corneum due to a decreased yield stress. As 590 expected, the pierced hole has a greater effect on the penetration depth. The penetration depth rises from 5911149.58 to $1151.66 \mu \mathrm{m}$ when Adminpatch MN 1500 is used for the pressure ranging from 3 to 60 bar. In 592 addition, Figure 10 shows the penetration depth of $18 \mu \mathrm{m}$ diameter of stainless steel micro-particles is less 593 than $30 \mu \mathrm{m}$ diameter. The effect of the micro-particles size on the penetration depth is discussed in the 594 following section.

595 


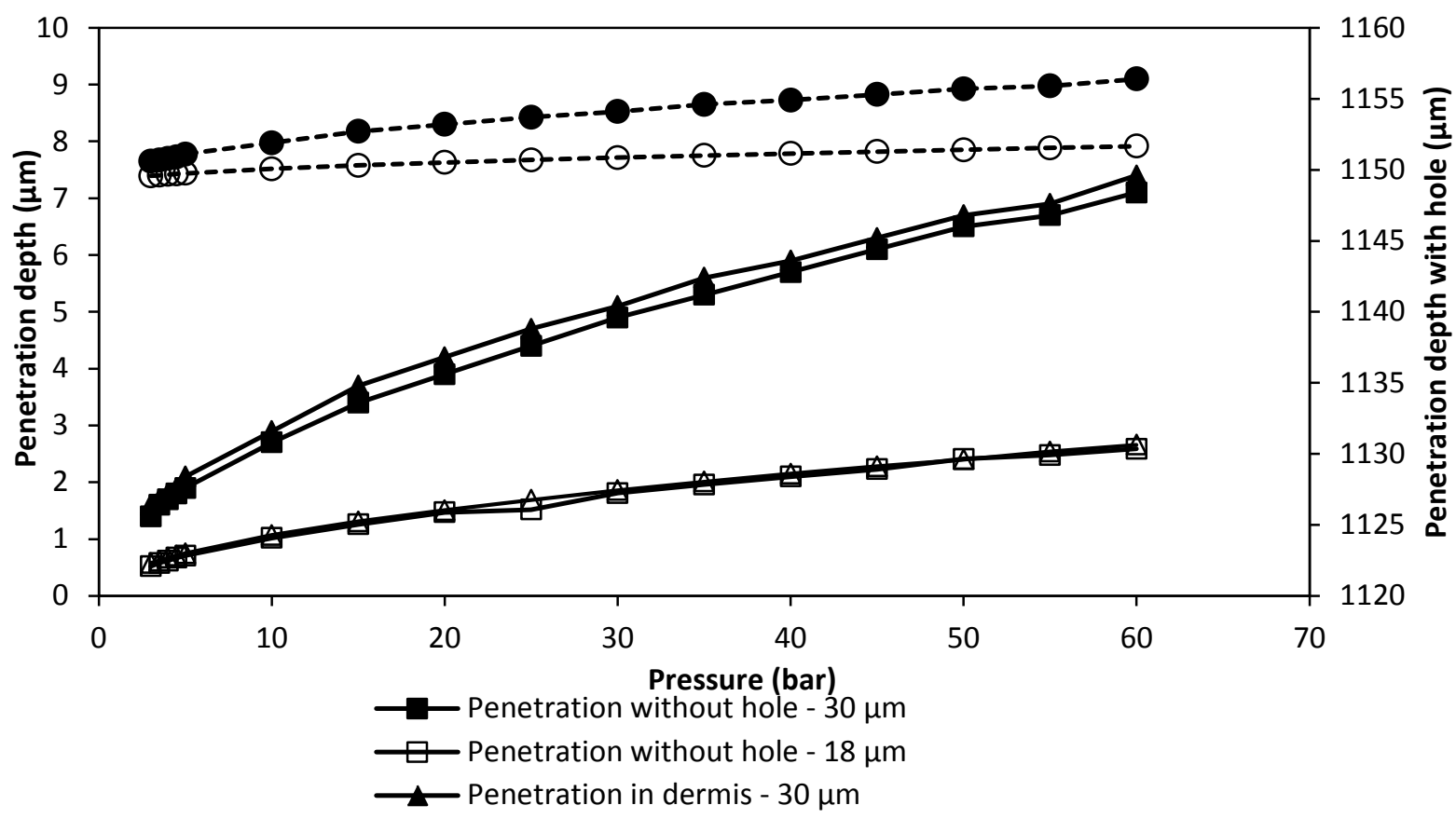

Figure 10: The effect of the operating pressure on the penetration depth of the micro-particles in the skin

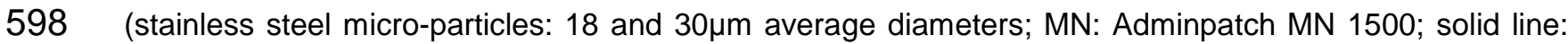

599 primary y axis; dashed line: secondary y axis)

600

601 The penetration depth of the micro-particles is also related to the size of the micro-particles which is one of

602 the major variables that affects the particle momentum. As presented in Figure 11, the diameter of the 603 micro-particles shows a positive correlation with the penetration depth. In this figure, the results plotted in 604 solid line are for the penetration of the micro-particles without pierced holes and are referred by the 605 primary y axis. The secondary y axis explains the results plotted in the dashed line and show the 606 maximum penetration depth of the micro-particles which goes through the pierced hole. The penetration 607 depth is found to be from 0.71 to $37.12 \mu \mathrm{m}$ in the top two layers of the skin (stratum corneum and viable 608 epidermis layers) at operating pressure of 5 bar while the particle diameter ranges from 18 to $140 \mu \mathrm{m}$. It 609 indicates that the small micro-particles cannot penetrate further in the skin so much as they are rebounded 610 by the skin due to the insufficient momentum. However, this condition may be fixed by the use of a MN 611 array. As shown in Figure 11, the penetration depth increases from 1149.75 to $1189.73 \mu \mathrm{m}$, which is 612 enhanced by delivering a number of micro-particles through the pierced holes created by the Adminpatch 613 MN 1500. The penetration depth in dermis layer varies from 0.75 to $40.73 \mu \mathrm{m}$ which is more than the 614 penetration in top layer. 


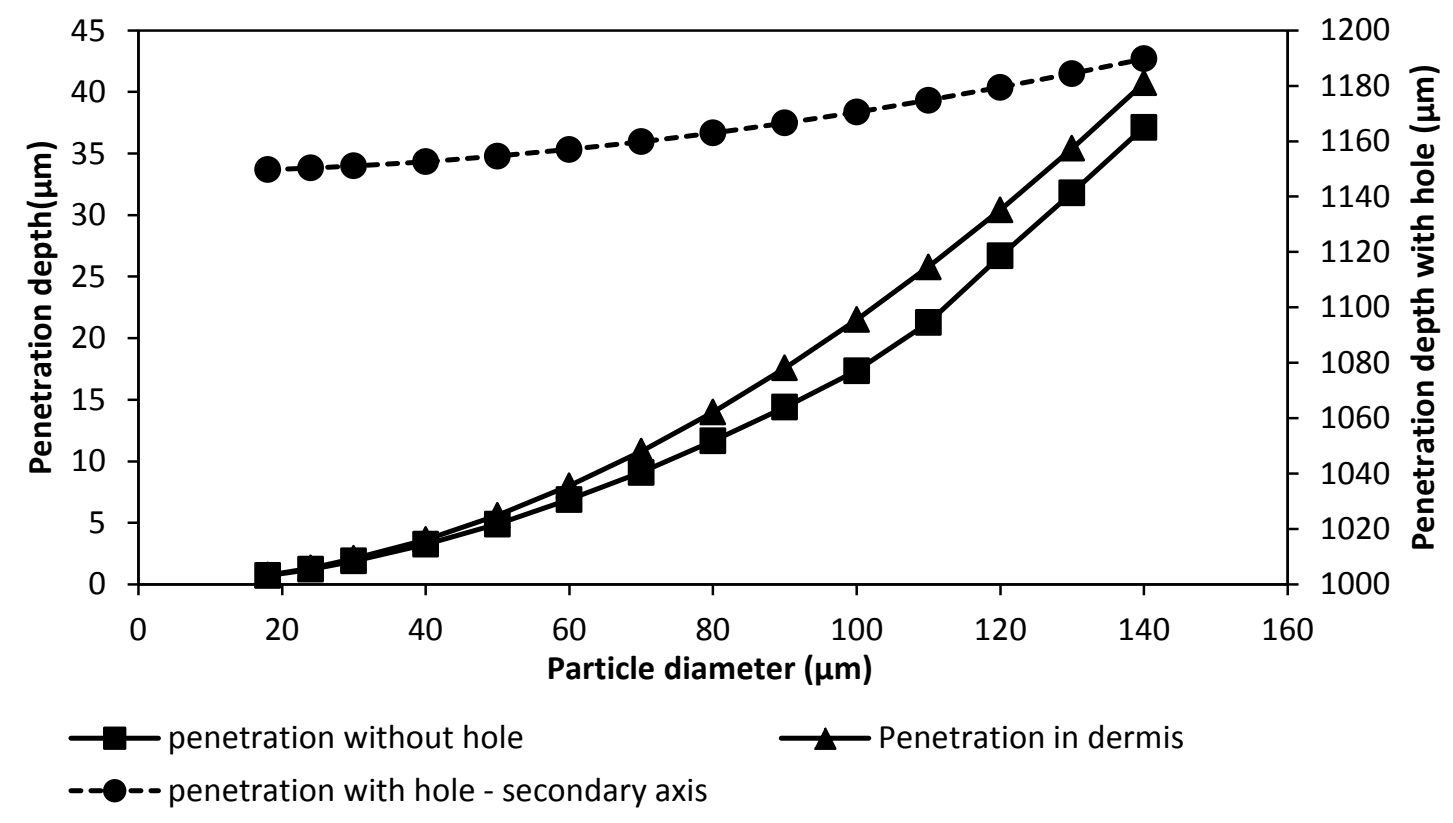

Figure 11: The penetration depth of stainless steel micro-particles inside skin against the particle diameter

617 (operating pressure: 5 bar; MN: Adminpacth MN 1500; solid line: primary y axis; dashed line: secondary y 618 axis)

619

620 A comparison between model and experimental results on penetration depths is shown in Figure 12.

621 Micro-particle penetration without (solid lines) and with (dashed lines) pierced holes refer to the primary

622 and secondary axes, respectively. As can be seen, the operating pressure and particle size have greater

623 effects on the penetration depth for the experimental results. The penetration depth rises fast as the

624 operating pressure is increased. It also shows a significant difference between stainless steel

625 micro-particles of 18 and $30 \mu \mathrm{m}$ diameter, which demonstrates that an increased particle size has a

626 positive correlation on the penetration depth. However, those two variables only have slight effects on the

627 penetration depth according to the model result. This is because the pellet is considered to be separated

628 into individual particles perfectly in the mathematical model which does not happen in practice. Zhang et al.

629 (2013b) have used different pore sizes of meshes to break up the pellet separation and control the size

630 distribution of the separated particles, which show that the pellet has been broken into individual particles

631 effectively with only a few small agglomerated particles. These agglomerated particles may cause an

632 increased penetration depth and further improve the effect of the operating pressure on the penetration. 


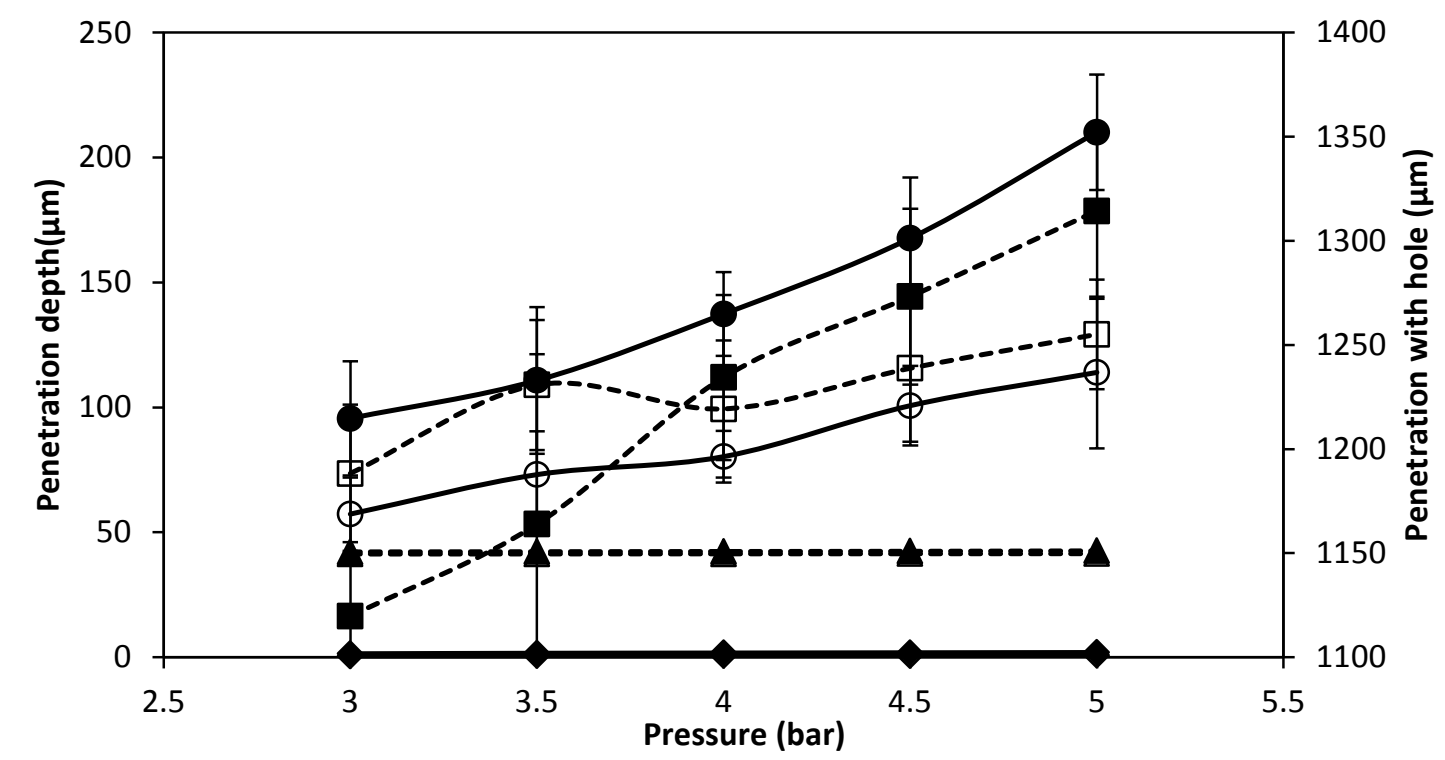

- Penetration without needle hole - $30 \mu \mathrm{m}$ (experimental)

$\neg$ Penetration without needle hole - $30 \mu \mathrm{m}$ (model)

- Penetration without needle hole - $18 \mu \mathrm{m}$ (experimental)

$\neg$ Penetration without needle hole - $18 \mu \mathrm{m}$ (model)

- - Penetration through needle hole - $30 \mu \mathrm{m}$ (experimental)

--1-- Penetration through needle hole - $30 \mu \mathrm{m}$ (model)

- $\square--$ Penetration through needle hole - $18 \mu \mathrm{m}$ (experimental)

-- - --Penetration through needle hole - $18 \mu \mathrm{m}$ (model)

635 Figure 12: A comparison between model and experimental results at various operating pressures

636 (stainless steel micro-particles: 18 and 30 diameters; MN: Adminpatch MN 1500; solid line: primary y axis;

637 dashed line: secondary y axis). The experimental results in the figure are generated from three repeats of

638 experiments. Due to possible formation of agglomerates in the experiments, experimental and the

639 modelling results do not match very well.

640

641 Figure 12 also shows that the micro-particles penetration through the pierced holes varies between model

642 and experimental results. For the model result, the length of the pierced holes is considered to be a

643 constant. Thus, the penetration difference between stainless steel micro-particles of 18 and $30 \mu \mathrm{m}$

644 diameters is only slight since the momentum of those two particles are insufficient to penetrate further in

645 the skin. These differ with experimental results which show that the penetration depth varies at the

646 operating pressure ranges from 3 to 5 bar. This is because the length of the pierced holes is varied after

647 the removal of the MNs in the experiment. It directly affects the micro-particles penetration depth, such as

648 small particles may penetrate further than larger particles. As presented in Figure 12, the penetration

649 depth of stainless steel micro-particles of $18 \mu \mathrm{m}$ diameter is more than $30 \mu \mathrm{m}$ diameter at 3 and 3.5 bar

650 pressure. However, the experimental results show that the stainless steel micro-particles of 30 and $18 \mu \mathrm{m}$

651 diameters reach the penetration depth from 1119.7 to $1314.4 \mu \mathrm{m}$ and from 1188.3 to $1255.1 \mu \mathrm{m}$ while the 
pressure varies from 3 to 5 bar, respectively. The operating pressure only presents a slight effect at this condition. As expected, the length of the pierced holes becomes the primary factor which maximizes the

654 penetration depth. It directly relates to the length of MN. The effect of the micro-needle length on the 655 penetration depth is discussed in the following section.

656

\subsubsection{The effect of the micro-needle length}

658 In principle, the length of the pierced hole depends on the length of the micro-needle. An increased length of MNs causes an increase in the pierced holes and thereby increases the penetration depth of micro-particles. As presented in Figure 13, the penetration depths of the micro-particles differ significantly between each application of MNs. Both model and experimental results present that the penetration depth increases from an increase of MN length. For the model result, the operating pressure does not show a great effect on the penetration depth. For the experimental result, the penetration depth is varied at the operating pressure ranges from 3 to 5 bar. This is because the effect of the agglomerates and the unmaintainable length of pierced holes, which are presented in previous section. The operating pressure presents a positive effect on the penetration depth, which agrees with the model result.

In conclusion, the experimental results match well with the model results in Figure 13. It confirms that this mathematical model is suitable for modelling MNs assisted micro-particles delivery. It also indicates that the micro-particles can be deposited at a desired depth in a target based on a use of specific lengths of

671 MNs. In addition, the penetration depth gradually increases with the increase in operating pressure. It can

672 be considered that based on the assistance of the holes on the micro-particles delivery, the penetration depth can be fine tuned by the operating pressure.

\section{$675 \quad 3.3$ Further discussions}

676 In this paper, the penetration depths of micro-particles are analyzed with respect to variations in operating 677 pressure, particle size, MN size using modelling and experimental results. It is evident that the particle 678 penetration depth increases from an increase of the operating pressure and particle size as the particle 679 momentum is related to those two key variables. In the experiments, the agglomerates provide a greater 680 effect on the particle penetration depth. This is possibly why the model results could not match well with 681 the experimental results in this paper. However, the agglomerates can be prevented by using a smaller 682 pore size of mesh to allow individual micro-particles to pass through which provide obtain a uniform penetration depths of the particles and comparable well with model results. 


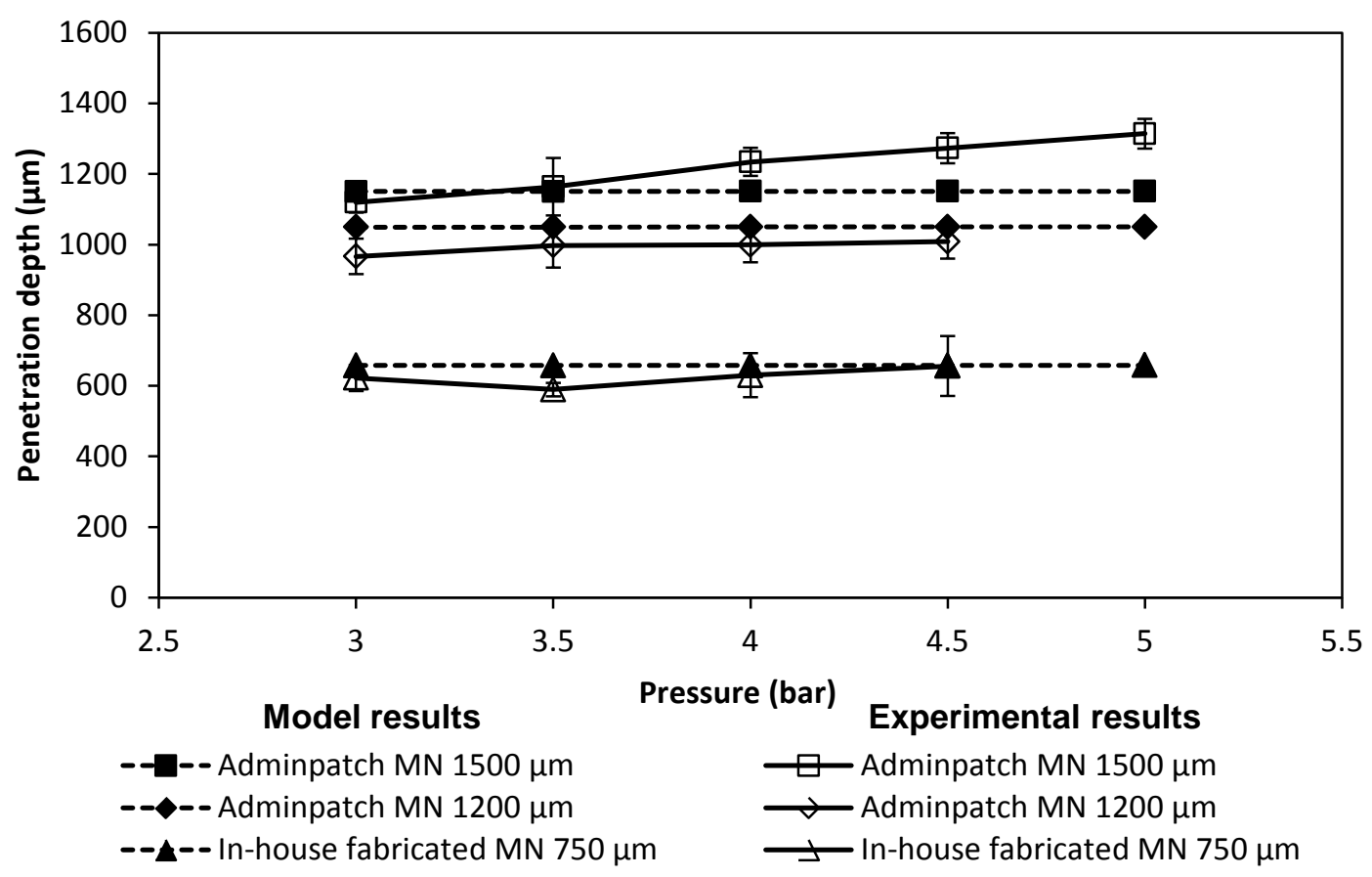

Figure 13: The effect of the micro-needle length on the penetration depth of the stainless steel micro-particles $(30 \mu \mathrm{m})$. The experimental results in the figure are generated from three repeats of experiments.

689 The paper confirms that an application of MN array provides a positive effect on the micro-particles 690 penetration. The maximum penetration depth of the micro-particles has presented a significant increment 691 from the results without MN application. For the MN assisted micro-particles delivery, the penetration 692 depth reaches the dermis layer inside skin, which was not achieved in the previous gene gun systems 693 (e.g., injection jet, light gas gun, contoured shock tube, etc). Mitchell et al. (2003) have used a light gas to 694 accelerate the stainless steel micro-particles of $25 \mu \mathrm{m}$ diameter to a velocity of $170 \mathrm{~m} / \mathrm{s}$ (20 bar) and 695 penetrate $150 \mu \mathrm{m}$ into excised canine buccal mucosa. Kendal (2001) has suggested that the $1 \pm 0.2 \mu \mathrm{m}$ 696 diameter gold particles can reach a velocity of $580 \pm 50 \mathrm{~m} / \mathrm{s}$ at 40 bar pressure using a contoured shock 697 tube and penetrate $66 \mu \mathrm{m}$ in the skin. The epidermis has been normally considered as the target tissue for 698 gene loaded particle delivery as the devices may be limited by the penetration depth they achieve (Trainer 699 et al., 1997). Now the target tissue may be the dermis layer as the use of MNs promise to increase the 700 penetration depths further. In other words, the MN may be useful for the injection of micro-particles especially for the targets which require a deeper injection of the particles. 
704 A mathematical model has been developed for MN assisted micro-particles delivery from gene guns. MN 705 assisted micro-particles delivery, and in particular the penetration depths of the particles, are studied in 706 this paper. For the acceleration stage, the particle velocity is analysed in relation to the operating pressure 707 and these results compare well with the experimental results obtained from the previous work (Zhang et 708 al., 2013b). For the deceleration stage, an individual micro-particle trajectory has been simulated in the 709 model. Additionally, the distribution of the micro-particles in three different layers has been determined 710 using modelling results. These results show that about $75 \%$ of particles penetrate into the stratum 711 corneum without going through the holes, and 23 and $2 \%$ of particles penetrate into epidermis and dermis layers via the pierced holes, respectively. The presented model for MN assisted micro-particles delivery

713 takes into consideration possible change in operating pressure, particle size, MN length due to the 714 micro-particles delivery is directly related to those key variables. Model results obtained indicate that 715 increasing the operating pressure and particle size would increase the penetration depth of the 716 micro-particles inside skin due to the increased momentum. In addition, the hole length is shown to be a 717 major variable which maximizes the particle penetration depth. The model results match well with the 718 experimental results for the penetration depth of micro-particles. In conclusion, the presented model is 719 shown to be useful for simulating micro-particle trajectories and penetration depth for MN assisted 720 micro-particles delivery.

721

722

\section{Acknowledgement}

723 Loughborough University (UK) is acknowledged for providing a PhD studentship to Dongwei Zhang which 724 made this work possible. Furthermore, the technical supports from Mr Tony Eyre, Mr Mark Barron, Mr Jim 725 Muddimer, Mr Terry Neale and Mr Steve Bowler are gratefully acknowledged.

726

\section{6. Conflicts of Interest}

728 The authors declare no conflict of interest

729

\section{Reference}

731 Al-Qallaf, B., Das, D.B., Davidson, A., 2009. Transdermal Drug Delivery by Coated Microneedles:

732 Geometry Effects on Drug Concentration in Blood, Asia-Pacific Journal of Chemical Engineering 4(6), 733 845-857, DOI: 10.1002/apj.353.

735 Alexander, H., Cook, T., 2006. Variations with age in the mechanical properties of human skin in vivo, 736 Journal of Tissue Viability 16(3), 6-11 
738 Bellhouse, B.J., Sarphie, D.F., Greenford, J.C., 1994. Needless syringe using supersonic gas flow for 739 particle delivery. Intternational Patent W094/24263

740

741 Bellhouse, B.J., Bell, J., Millward, H.R., Phillips, M.J., Nabulsi, S.M., 2003. Particle delivery, US Patent US 7426592545 B1

743

744 Bellhouse, B.J., Greenford, J.C., Sarphie, D.F., 2006. Particle delivery, European Patent EP 1637173 A2 745

746 Chen, D., Maa, Y.F., Haynes, J.R., 2002. Needle-free epidermal powder immunization, Expert. Review of 747 Vaccines 1(3), 265-276

748

749 Cheung, K, Han, T, Das, DB, 2014. Effect of Force of Microneedle Insertion on the Permeability of Insulin 750 in Skin, Journal of Diabetes Science and Technology, 8(3), 444-452, DOI: 10.1177/1932296813519720. 751

752 Crozier, W.D., Hume, W., 1957. High velocity light gas gun, Journal of Applied Physics 28(8), 892-895 753

754 Dehn, J., 1987. A Unified theory if penetration, International journal of impact engineering 5, 239-248 755

756 Diridollou, S., Black, D., Lagarde, J.M., Gall, Y., Berson, M., Vabre, V., Patat, F., Vaillant, L., 2000. Sex757 and site-dependent variations in the thickness and mechanical properties of human skin in vivo, 758 International Journal of Cosmetic Science 22(6), 421-435

759

760 Donnelly, R.F., Garland, M.J., Singh, T.R.R., Migalska, K., Majithiya, R., McCrudden, C.M., Kole, P.L., 761 Mahmood, T.M.T., McCarthy, H.O., Willfson, A.D., 2012. Hydrogel-Forming microneedle array for 762 enhanced transdermal drug delivery, Advanced Functional Materials, 22, 4879-4890

763

764 Duck, F.A., 1990. Physical Properties of Tissue: A Comprehensive Reference Book, Academic Press, 765 Harcourt Brace Jovanovich, London

767 Eberly, D., 2002. Rotation representations and performance issue, Geometric Tools, LLC 768 (http://www.geometrictools.com/Documentation/Rotationlssues.pdf) [Accessed on 3/6/2013] 
770 Han, T., Das, D.B., 2013. Permeability enhancement for transdermal delivery of large molecule using

771 low-frequency sonophoresis combined with microneedles, Journal of Pharmaceutical Science 102(10),

$772 \quad 3614-3622$

773

774 Hardy, H.P., Quinlan, N.J., Kendall, M.A.F., Bellhouse, B.J., 2001. Numerical study of a prototype 775 needle-free powdered vaccine and drug delivery system. In: ISSW 23, Austin, TX, USA, 23-37

776

777 Holbrook, K. A., Odland, G.F., 1974. Regional Differences in the thickness (cell layers) of the human 778 stratum corneum: an ultrasound analysis, Journal of Investigative Dermatology, 62, 415-422.

779

780 ImageJ, 2013. ImageJ (http://rsbweb.nih.gov/ij/disclaimer.html) [Accessed on 3/6/2013]

781

782 Kendall, M.A.F., Carter, F.V., Mitchell, T.J., and Bellhouse, B.J., 2001. Comparison of the transdermal 783 ballistic delivery of micro-particle into human and porcine skin, Proceedings of the 23rd Annual 784 International Conference of the IEEE, 2991-2994

785

786 Kendall, M.A.F., Quinlan, N.J., Thorpe, S.J., Bellhouse, B.J., Ainsworth, R.W. 2004a. Measurements of 787 the gas and particle flow within a converging-diverging nozzle for high speed powdered vaccine and drug delivery, Experiments in Fluids 37, 128-36

789

790

Kendall, M.A.F., Rishworth, S., Carter, F., Mitchell, T., 2004b. Effects of relative humidity and ambient 791 temperature on the ballistic delivery of micro-particles to excised porcine skin, Journal of Investigative 792 Dermatology $122,739-746$

793

794 Kishino, A., Yanagida, T., 1988. Force measurements by micromanipulation of a single actin filament by 795 glass needles, Nature 334, 74-76

796

797 Lambert, P.H., Laurent, P.E., 2008. Intradermal vaccine delivery: Will new delivery systems transform 798 vaccine administration?. Vaccine 26, 3197-3208.

799

800 Lin, M., 2000. The gene gun: current applications in cutaneous gene therapy, International Journal of 801 Dermatology 39, 161-170

802 
Liu, Y., 2006. "Physical-mathematical modeling of fluid and particle transportation for DNA vaccination",

804 International Journal of Engineering Science 44(15-16), 1037-1049

805

806 Liu, Y., (2007). "Impact studies of high-speed micro-particle following biolistic delivery", IEEE Transaction 807 on Biomedical Engineering, 54(8), 1507-1513

808

809

Matteucci, M., Fanetti, M., Casella, M., Gramatica, F., Gavioli, L., Tormen, M., Grenci, G., De Angelis, F.,

810 Di Fabrizio, E., 2009. Poly vinyl alcohol re-usable masters for MN replication. Microelectronic Engineering,

811 doi:10.1016/j.mee.2009.01.068

812

813 McAllister, D.V., Wang, P.M., Davis, S.P., Park, J.H., Canatella, P.J., Allen, M.G., Prausnitz, M.R., 2003.

814 Microfabricated needles for transdermal delivery of macromolecules and nanoparticles: fabrication

815 methods and transport studies. Proceedings of the National Academy of the Sciences of the United States

816 of America 100, 13755-13760.

817

818 Mitchell, T.J., Kendall, M.A.F., Bellhouse, B.J., 2003. A ballistic study of micro-particle penetration to the 819 oral mucosa, International Journal of Impact Engineering 28, 581-599

820

821 Morsi, S.A., Alexander, A.J., 1972. An investigation of particle trajectories in two-phase flow systems, 822 Journal of Fluid Mechanics 55(2), 193-208

823

824 Nayak, A., Das, D.B., 2013. Potential of biodegradable microneedles as a transdermal delivery vehicle for 825 lidocaine, Biotechnology Letters 35(9), 1351-1363

826

827 Nayak, A., Das, D.B., Vladisavljević, G.T., 2013. Microneedle-Assisted Permeation of Lidocaine 828 Carboxymethylcellulose with Gelatine Co-polymer Hydrogel. Pharmaceutical Research 31(5), 1170-1184 829

830 O'Brien, J.A., Lummis, S.C.R., 2006. Biolistic transfection of neuronal cultures using a hand-held gene 831 gun, Nature Protocols 1(2), 977-981

832

833 Parker, F., 1991. Structure and Function of the Skin: Paper 1 in Dermatology edited by Orkin, M. and 834 Maibach, H. I. and Dahl, M. V., 1st edition (Norwalk, Connecticut: Appleton and Lange). 
Phipps, J. B., Padmanabhan, R. V., Lattin, G. A., 1988. Transport of Ionic Species Through Skin. Solid

837 State Ionics 28, 1778-1783.

838

839 Pierard, G.E., Lapiere, C.M., 1977. Physiopathological variations in the mechanical properties of skin,

840 Archives of Dermatological Research 260(3), 231-239

841

842 Olatunji, O., Das, D.B., 2010. Painless Drug delivery using microneedles. Current technologies to increase

843 the transdermal delivery of drugs (Editor: Joan Escober Chavez). Bentham Science Publishers (available

844 online at http://www.benthamdirect.org/pages/b_getarticlebybook.php). ISBN:978-1-60805-191-5

845

846 Olatunji, O., Das, D.B., 2011. Drug delivery using Microneedles. Comprehensive Biotechnology (Editor:

847 Zhanfeng Cui). 2nd Edition, MS number 501. MRW, Elsevier, The Boulevard, Langford Lane, Oxford,

848 United Kingdom. ISBN: 13: 978-0-444-53352-4

849

850 Olatunji, O., Das, D.B., Nassehi, V., 2012. Modelling transdermal drug delivery using microneedles: Effect 851 of geometry on drug transport behaviour, Journal of Pharmaceutical Sciences 101(1), 164-175,

852

853 Olatunji, O., Das, D.B., Garland, M.J., Belaid, L., Donnelly, R.F., 2013. Influence of Array interspacing on 854 the force required for successful microneedle skin penetration: theoretical and practical approaches, 855 Journal of Pharmaceutical Science 102(4), 1209-1221.

856

857 Olatunji, O, Igwe, CC, Ahmed, AS, Alhassan, OA, Asieba, GO, Das, DB, 2014. Microneedles from fish 858 scale biopolymer, Journal of Applied Polymer Science, 131(12), DOI: 10.1002/app.40377.

859

860 Quinlan, N.J., Kendall, M.A.F., Bellhouse, B.J., Ainsworth, R.W., 2001. Investigations of gas and particle 861 dynamics in first generation needle-free drug delivery device, Shock Waves 10, 395-404

862

863 Rushmer, R. F., Buettner, K. J. K., Short, J. M., Odland, G. F., 1966. The Skin, Science 154, 343864348.

865

866 Schaefer, H., Redelmeier, T.E., 1996. Skin barrier: Principles of percutaneous absorption, Karger, New 867 York

868 
871 Shampine, L.F., Reichelt, M.W., 1997. The matlab ode suite, SIAM Journal on Scientific, Computing 18(1), $872 \quad 1-22$

873

874 Soliman, S.M., Abdallah, S., Gutmark, E., Turner, M.G., 2011. Numerical simulation of microparticles 875 penetration and gas dynamics in an axi-symmertric supersonic nozzle for genetic vaccination, Powder 876 Technology 208, 676-783

877

878 Trainer, A.T., Alexander, M.Y., 1997. Gene delivery to the epidermis, Human Molecular Genetics, 6(10), $8791761-1767$

880

881 Truong, N.K., Liu, Y., Kendall, M.A.F., 2006. Gas and particle dynamics of a contoured shock tube for 882 pre-clinical micro-particle drug delivery, Shock Waves 15, 149-164

883

884 Webster, C., 1995. The Discovery of Boyle's law, and the concept of the Elasticity of Air in the 885 Seventeenth Century. Archive History Exact Sciences 2, 441-502

886

887 Wildnauer, R.H., Bothwell, J.W., Douglas, A.B., 1971. Stratum corneum properties I. Influence of relative 888 humidity on normal and extracted stratum corneum, Journal of Investigative Dermatology, 56, 72-78

889

890 Yager, E.J., Stagnar, C., Gopalakrishnan, R., Fuller, J.T., Fuller, D.H., 2013. Optimizing particle-mediated 891 epidermal delivery of an influenza DNA vaccine in ferrets, Methods in Molecular Biology 940, 223-237

892

893 Yang, C.H., Shen, S.C., Lee, J.C., Wu, P.C., Hsueh, S.F., Lu, C.Y., Meng, C.T., Hong, H.S., Yang, L.C., 894 2004. Seeing the gene therapy: application of gene gun technique to transfect and decolour pigmented rat 895 skin with human agouti signalling protein cDNA, Gene Therapy 11(13), 1033-1039.

896

897 Zhang, M.J., Tao, W.M., Pianetta, P.A., 2007. Dynamics modeling of biolistic gene guns, Physics in 898 Medicine and Biology 52,1485-1493.

899 
901 gene guns: A review. Drug Delivery, DOI: 10.3109/10717544.2013.864345 (in press).

902

903 Zhang, D.W., Das, D.B, Rielly, C.D., 2013b. An experimental study of microneedles assisted

904 micro-particle delivery using a model system. Journal of Pharmaceutical Science 102(10), 3632-3644

905

906 Zhang, D.W., Das, D.B., Rielly, C.D. 2014. Microneedles assisted micro-particles delivery from gene guns:

907 Experiments using skin mimicking agarose gel. Journal of Pharmaceutical Sciences 103(2), 613-627.

908

909

910

911

912

\section{Appendix}

The drag coefficient as function of particle Reynolds number, as used in this work (Morsi and Alexander, 1972)

913

$914 C_{d}=24.0 / \mathrm{Re}$

for $\operatorname{Re}<0.1$

915

$C_{d}=22.73 / \mathrm{Re}+0.0903 / \mathrm{Re}^{2}+3.69$

for $0.1<\operatorname{Re}<1.0$,

916

$C_{d}=29.1667 / \mathrm{Re}-3.8889 / \mathrm{Re}^{2}+1.222$

for $1.0<\operatorname{Re}<10.0$

917

$C_{d}=46.5 / \mathrm{Re}-116.67 / \mathrm{Re}^{2}+0.6167$

for $10.0<\operatorname{Re}<100.0$

918

$C_{d}=98.33 / \mathrm{Re}-2778 / \mathrm{Re}^{2}+0.3644$

for $100.0<\operatorname{Re}<1000.0$

919

$C_{d}=148.62 / \operatorname{Re}-4.75 \times 10^{4} / \mathrm{Re}^{2}+0.357$ for $1000.0<\mathrm{Re}<5000.0$ 